MODELING, IDENTIFICATION AND CONTROL, 1997, VOL. 18, NO. 1, 19-59

doi:10.4173/mic.1997.1.2

\title{
Process Modelling of Chemical Reactors: Zero- versus Multi-dimensional Models
}

\section{BJØRN H. HJERTAGER}

Keywords: Multiphase, multidimensional, gas/liquid, gas/particle, bioreactor, fluidized bed

Trends in modelling of flow processes in chemical reactors are presented. Particular emphasis is given to models that use the multi-dimensional multi-fluid techniques. Examples are given for both gas/liquid as well as gas/particle reactors.

\section{Introduction}

\subsection{General}

Many different processes influence the total performance characteristics of chemical reactors. Among these are the gas/particle or gas/liquid or gas/liquid/particle flow and distribution, the chemical reactions and the heat transfer. All of these processes occur simultaneously and are intimately coupled to each other. The performance of the reactor is strongly dependant on the interactions between these processes and the geometrical design of the reactor. Because of the complexities of such problems there are no analytical methods available that can describe in detail what is occurring inside a chemical reactor. In each new reactor it is very often the geometrical details that will determine the performance of the final design of the reactor. Until recently the design of chemical reactors has therefore been based on experimental work done in laboratory and pilot plant scale and extrapolated these data to full scale by application of various empirical scaling techniques.

These design techniques may be very expensive and give insufficient and inaccurate information about the performance.

\subsection{Model types}

Process models of reactors have until recently been limited to zero-dimensional models. In these models total and species mass balances have been set up with the whole reactor volume as control surface. Figure 1a gives an example of the control surface for a stirred reactor. The conservation equation for a chemical species is given by:

$$
\begin{aligned}
\frac{d}{d t}\left(\rho V Y_{j}\right) & =\rho_{i} F_{i} Y_{i j}-\rho_{o} F_{o} Y_{o j}+\quad V R_{j} \\
{[\text { accumulation }] } & =[\text { inflow }]-[\text { outflow }]+[\text { net generation }]
\end{aligned}
$$

The balances include the terms containing accumulation, net inflow and net generation rate of the species in question. The main assumption in this model is that there are no gradients in the dependent variables (i.e. concentrations). No momentum

Received 9 July 1996.

An early version of this paper was presented at the SIMS '96 Applied Modelling and Simulation Conference, Trondheim, Norway, June 11-13 1996.

Department of Process Technology (IPT), Telemark Institute of Technology (HiT-TF) and Telemark Technological R\&D Centre (Tel-Tek), Kjølnes Ring, N-3914 Porsgrunn, Norway. 

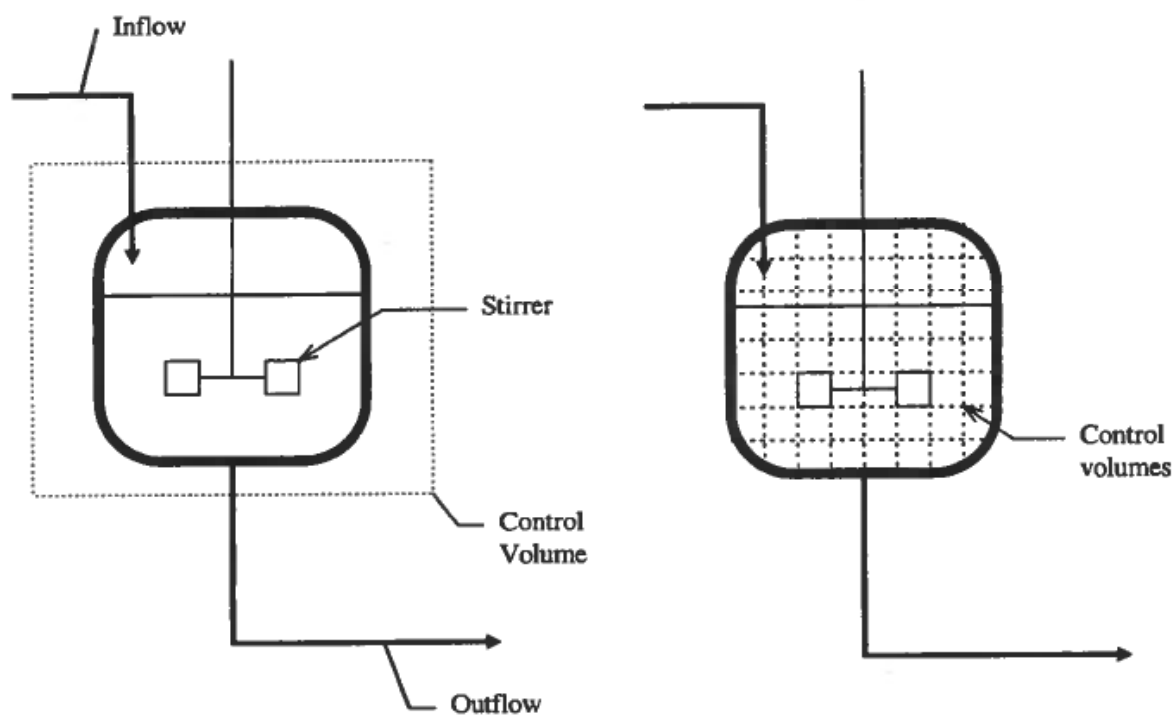

Figure 1. Schematic view of a stirred reactor with control surfaces for a) zero-dimensional and b) multi-dimensional models.

balances are needed for this type of model. The consequence of this model is that a set of ordinary differential equations have to be solved.

It is only during the last few decades that $2 \mathrm{D}$ and $3 \mathrm{D}$ models have been developed. These multi-dimensional models set up balance equations for sub-domains (computational cells or control volumes) in the reactor volume. As for the zero-dimensional models total and species mass balance have to be set up for each of these sub-domains. Figure $1 \mathrm{~b}$ gives an example of the set of non-overlapping control surfaces that are used for these models and the conservation equation for a chemical species reads:

$$
\begin{aligned}
\frac{\partial}{\partial t}\left(\rho Y_{j}\right) & =-\nabla \cdot\left(\rho \vec{V} Y_{j}\right)-\nabla \cdot \vec{J}_{Y j}+R_{j} \\
{[\text { accum. }] } & =[\text { net inflow }]+[\text { net diffusion }]+[\text { net generation }]
\end{aligned}
$$

In addition, the flow in and out of the sub-domains must also be calculated. For this we need to put up momentum balances. One momentum balance has to be set up for each direction, i.e. two for $2 \mathrm{D}$ and three for $3 \mathrm{D}$. The new process that has to be included is diffusion of the conserved quantity in question. This is due to the fact that concentrations are not homogeneous inside the reactor. This means that in addition to accumulation, net inflow and net generation, net diffusion needs to be modelled. In order to model this diffusion, gradient transport is assumed with an effective transport coeffisient (diffusion coeffisient for species transport and viscosity for momentum transport). Since the flow is turbulent this effective transport coefficient is dependent on characteristics of the turbulence. Two parameters are decisive for describing the state of turbulence, namely local turbulence velocity and length scale of the turbulent eddies. This has led to the so-called two-equation turbulence model (Launder and Spalding (1974)) for gas/liquid and the granular theory (Gidaspow (1994)) for gas/particle flows. These models are deduced by setting up conservation equations for two quantities. The most popular of these models for gas/liquid are named the $\mathrm{k}-\varepsilon$ model, where $\mathrm{k}$ is the turbulent kinetic energy and $\varepsilon$ is the rate of dissipation of turbulent kinetic energy. From 
$\mathrm{k}$ and $\varepsilon$ we may deduce the turbulence velocity and length scale and from them the effective transport coefficient. A general feature of the multi-dimensional models is that we need to solve a set of partial differential equations. This means that special care must be taken to obtain efficient means of solving these equations (Patankar (1980), Spalding (1985)). Special treatments are needed for impeller and baffle regions in mechanically stirred reactors.

\subsection{Objective of the paper}

The aim is to present multidimensional prediction/design models for the chemical reaction and transport processes in multi-phase flow of gas/liquid and gas/particle chemical reactors.

Emphasis will be given to models of the finite-domain type in which the governing equations are integrated over discrete volumes inside the reactor. A multi-fluid approach will be described to enable modelling of the processes that occur in the various phases involved as well as the transfer between the phases. Models of turbulence, agitators, heat and mass transfer will also be discussed. Finally, some examples of flow and chemical reaction modelling of bioreactors and fluidized bed reactors will be given.

\section{Review of multi-dimensional multi-phase models}

\subsection{Background and motivation}

General

The new multi-dimensional computer techniques for fluid flow processes have now made it possible to develop new design methods based on mathematical modelling of the basic processes that occur in the actual geometrical model of the reactor. To apply these methods the basic conservation equations of flow, chemical reactions and heat transfer as well as the coupling between them and the coupling between the phases must be solved for a multi-dimensional calculation domain.

\section{Gas/liquid reactors}

Many production processes based on biotechnology depend on oxygen-requiring microorganisms in suspension. Gas-liquid bioreactors form the core of all such processes. A bioreactor designer has to ensure that the reactor provides for: 1) adequate oxygen transfer, 2) sufficient agitation and mixing and 3) can handle the heat transfer loads, at acceptable capital and operating costs.

The oxygen needs are often the most difficult to satisfy. In theory, there should be no difficulty in meeting these demands, were it not for the special constraints encountered in bio-processing: the oxygen solubility in the typical aqueous media is low; the biological broths can be highly viscous and have non-Newtonian behaviour; the low shear tolerance and susceptibility to mechanical damage of the biological catalyst.

These limitations often lead to poor mixing and mass transfer in bioreactors. Furthermore, the restrictions on acceptable shear levels are becoming more tight with increasing use of cell cultures in commercial production.

Reliable assessments of performance and scale-up of bioreactors will require more detailed understanding and characterization beyond the scope of traditional empirical approaches in process design, i.e. global performance correlations obtained in replica modelling tests. The research needed to achieve this goal will involve experimental 
investigations and mathematical modelling of the local processes encountered by organisms flowing through the non-homogeneous turbulent and bubbly flow fields.

\subsection{Previous work}

\section{Gas/particle reactors}

Gidaspow (1986) has reviewed the CFD models for gas/particle reactors that had evolved up until about 1985 . The models at that time had demonstrated the capabilities to simulate some of the crucial characteristics like the bubble formation and development in bubbling fluidized beds. The models assumed a two-phase gas-particle flow with given apparent viscosities in the gas and in the particle phases. Shih $\boldsymbol{e t}$ al. (1987) introduced a model that takes account of the multi-sized particle problem and showed application to a sedimentation problem. Mathiesen et al. (1996) have used a multisize model to analyze segregation in a riser. The work of Tsuo and Gidaspow (1990) showed that the model was able to calculate the flow patterns in circulating fluidized beds. This model had to assume an apparent viscosity for the particle phase to be able to predict the experimentally observed phenomena. The works of Ding and Gidaspow (1990) and Ma and Ahmadi (1990) propose to couple the apparent viscosities in the two phases by introducing turbulence models for each of the phases. The works of Samuelsberg et al. (1996a, b) have continued this work. A comprehensive model for two phase flow, heat transfer and chemical reactions in a fluidized bed gasifier has been presented by Gidaspow et al. (1985). Manger et al. (1995a and b, 1996a and b) have formulated the multi-phase flow equations in curvilinear coordinates enabling analyses of complex geometries.

A variety of models have been proposed in order to design catalytic commercialscale fluidized bed reactors from experimental findings in small scale units. In order to verify these models, well-known catalytic reactions were run in both small and relatively large scale units. Almost all of these experiments were carried out in small scale bubbling fluidized bed reactors. To the author's knowledge, relatively few studies have been reported which uses multi-phase CFD models to simulate chemical reactions in catalytic fluidized bed reactors. Gidaspow et al. (1985) studied the IGT-U-GAS coal gasification process and predicted many of the features of fluidized bed gasifiers. Theologos and Markatos (1992 and 1993) used a three dimensional model to simulate a catalytic cracking riser type reactor. With a simplified kinetic model they simulated among other variables the yields and temperature distribution inside the reactor. Gidaspow and Therdthianwong (1993) studied the effect of gas velocity of $\mathrm{SO}_{2}$ sorption with calcined limestone in an isothermal CFB loop. Samuelsberg and Hjertager (1995) simulated an isothermal ozone decomposition reaction in a circulating fluidized bed reactor. The predictions were compared against available ozone decomposition data. The predicted radial and axial ozone concentration profiles fitted the experimental data well, in both shape and magnitude. Samuelsberg and Hjertager (1996) have analyzed an exothermal oxy-chlorination reaction in a large scale bubbling bed reactor.

\section{Gas/liquid reactors}

The basis for general prediction models of bioprocesses in bioreactors is the modelling of turbulent two-phase flow in these reactors. Issa and Gosman (1981) simulated the two-phase flow in a baffled, agitated vessel. They used a 3-D model, with a simplified equation for the gas momentum, and the k- $\varepsilon$ turbulence model. Pericleous and Patel (1987) modelled the flow in stirred vessels, using an algebraic slip model. 
Momentum equations were solved using mixture properties. The spatial distribution of bubbles was calculated by the concentration equations, assuming constant bubble size. Experimental results and 3-D calculations of the flow processes occurring in a stirred reactor were presented by Bakker and van den Akker (1991). Single phase laser Doppler measurements were performed, and for gas-liquid flow, local gas fraction, bubble size and local oxygen mass transfer rate were measured. From a single phase calculation and assumptions of the phase slip, they calculated the distribution of gas in the vessel. Patterson (1991) used the LDA technique to measure liquid velocities with and without gas sparger. A 3-D calculation of the flow with the FLUENT code was applied, where the individual bubbles were tracked by a Langrangian approach. Bröring et al. (1991) and Fisher et al. (1992) used an improved ultrasound-Doppler technique for extensive measurements of bubble velocity components in stirred vessels equipped with three and one Rushton impeller, respectively. Gosman et al. (1992) calculated the gas-liquid flow in a stirred vessel with a 3-D calculation domain. The simulation results on local gas fraction were compared against experimental data. They used a two-fluid model and a k- $\varepsilon$ turbulence model, extended for two-phase flow. Trägårdh (1988) presented a 2D model of a mechanically stirred reactor. The bubble flow was described by a simplified model. The paper by Boysan et al. (1988) also applied a 2D model to an agitated vessel, whereas the model of Smith et al. (1988) presented preliminary results from a 3D model and single phase approximation of an agitated vessel with baffles. Morud and Hjertager (1996) have measured and simulated the gas liquid flow in a 15 litre stirred vessel. The bubble velocity measurements were done with a Laser doppler anemometer and the simulations used a two-dimensional two-fluid model. Trägărdh (1990) has further developed his model by introducing a two-scale turbulence model. Norman et al. (1993a, b and c) have introduced integrated models in which both the fluid dynamics and microbial kinetics are modelled.

The flow pattern in bubble columns has been analyzed by Torvik and Svendsen (1990) and Morud et al. (1991) using two-dimensional computational fluid dynamics models. The results of these studies showed reasonable agreement between model predictions and experiments. The model of Morud and Hjertager (1992) has also included submodels for the biochemical reactions that occur in bubble column bioreactors.

\section{Governing equations}

\subsection{General}

Two different approaches are available for setting up the governing equations for multi-phase flow in reactors. One method is the socalled PSIC (Particle-Source-In-Cell) procedure originally presented by Migdal and Agosta (1967). This method treats the continuous phase (liquid or gas) in a usual Eulerian description, whereas the dispersed phase (bubbles or particles) are described in a Langrangian way. This means that the dispersed phase is tracked through the flow domain from inlet to outlet. The presence of bubbles/particles are coupled to the continuous phase through sources of mass momentum and energy. This method may only be used for small values of the volume fraction of the dispersed phase.

The other method deduces the governing equations based on the Eulerian concept and is named the multi-fluid method (Spalding (1985)). This means that the phases are treated as interpenetrating fluids that share the space and interact with each other through the source terms. All equations are such that the volume fractions may take 
values between zero and one. The remainder of this paper will present a multi-fluid model.

\subsection{The multi-fluid model}

\section{General}

The numerical model of the turbulent two-phase flow is based on a two-fluid model (Spalding (1985)). Transport equations governing the conservation of mass, momentum and scalar variables like turbulent quantities ( $\mathrm{k}$ and $\varepsilon$ ) and concentration of chemical species are derived for each phase. The multi-fluid conservation equations governing the mean turbulent flow field are written as follows:

Mass:

$$
\frac{\partial}{\partial t}(\alpha \rho)_{k}+\nabla \cdot(\alpha \rho \vec{V})_{k}=0
$$

Momentum:

$$
\frac{\partial}{\partial t}(\alpha \rho \vec{V})_{k}+\nabla \cdot(\alpha \rho \vec{V} \vec{V})_{k}=-\nabla \cdot(\alpha \underline{\sigma})_{k}-\alpha_{k} \nabla p+\vec{F}_{k}+\alpha_{k} \rho_{k} \vec{g}
$$

Scalar:

$$
\frac{\partial}{\partial t}(\alpha \rho \Phi)_{k}+\nabla \cdot(\alpha \rho \vec{V} \Phi)_{k}=-\nabla \cdot\left(\alpha \vec{J}_{\Phi}\right)_{\mathrm{k}}+S_{\Phi}
$$

Here the subscript $\mathrm{k}$ refers to phase number $\mathrm{k}$. For a two-phase gas liquid flow $\mathrm{k}$ is either the gas (g) or the liquid (l) phase. For gas particle flows $\mathrm{k}$ is either gas (g) or particle size number, solid (s). The pressure, $\mathrm{p}$ is considered to be common for the various phases. Turbulent diffusive transport of momentum and scalar variables are denoted $\underline{\sigma}_{k}$ and $\vec{J}_{\Phi, \mathrm{k}}$, respectively. Interphase momentum transfer are denoted $\overrightarrow{\mathrm{F}}_{\mathrm{k}}$.

\subsection{Turbulence model}

\section{Gas/liquid}

The Reynolds stresses, $\underline{\sigma}$, in equation (4) and the turbulent scalar fluxes in equation (5) are related to the mean flow field through the Boussinesq approximation and are given by:

$$
\begin{gathered}
\underline{\sigma}_{k}=-\mu_{t}\left[\nabla \vec{V}_{k}+\left(\nabla \vec{V}_{k}\right)^{T}\right] \\
\vec{J}_{\Phi, k}=-\frac{\mu_{t}}{\sigma_{\Phi}} \nabla \Phi_{k}
\end{gathered}
$$

The Prandtl/Schmidt number $\sigma_{\Phi}$ is assumed to be constant, and the turbulent viscosity $\mu_{\mathrm{t}}$ is modelled using the two parameter k- $\varepsilon$ turbulence model (Launder and Spalding (1974)) as:

$$
\mu_{t}=c_{\mu} \rho_{k} \frac{k_{l}^{2}}{\varepsilon_{l}}
$$

For two-phase flows, the turbulence is commonly assumed to be a property of the continuous (liquid) phase (Torvik and Svendsen (1990)). The modelled transport equations for the turbulent energy $k_{1}$ and the dissipation rate $\varepsilon_{1}$ is therefore solved for the liquid phase:

$$
\left.\frac{\partial}{\partial t}(\alpha \rho k)_{l}+\nabla \cdot(\alpha \rho \vec{V} k)_{l}=\nabla \cdot\left(\alpha \frac{\mu_{l}}{\sigma_{k}}\right) \nabla k\right)_{l}+\alpha_{l}\left(G+P_{b}-\rho \varepsilon\right)_{l}
$$




$$
\frac{\partial}{\partial t}(\alpha \rho \varepsilon)_{l}+\nabla \cdot(\alpha \rho \vec{V} \varepsilon)_{l}=\nabla \cdot\left(\alpha \frac{\mu_{t}}{\sigma_{\varepsilon}} \nabla \varepsilon\right)_{l}+\alpha_{l} \frac{\varepsilon_{l}}{k_{l}}\left(c_{1}\left(G+P_{b}\right)-c_{2} \rho \varepsilon\right)_{l}
$$

The production of turbulence due to mean strain is given by:

$$
G=-\underline{\sigma}: \nabla \vec{V}
$$

Additional turbulent kinetic energy is produced due to the work induced by the bubbles when they move through the liquid phase:

$$
P_{b}=c_{i} \vec{F}_{l} \cdot\left(\vec{V}_{g}-\vec{V}_{l}\right)
$$

As in previous works (Lopez de Bertodano et al. (1990)) a value of 0.02 was used for $\mathrm{c}_{\mathrm{i}}$, which means that only about 2 percent of the bubble-induced turbulence goes into the large eddy structure of the continuous phase.

The values of the other constants applied in the k- $\varepsilon$ model are given in Table 1 .

Table 1. Values of constants in the k- $\varepsilon$ model.

\begin{tabular}{ccccc}
\hline $\mathrm{c}_{\mu}$ & $\mathrm{c}_{1}$ & $\mathrm{c}_{2}$ & $\sigma_{\mathrm{k}}$ & $\sigma_{\varepsilon}$ \\
\hline 0.09 & 1.44 & 1.92 & 1.0 & 1.3 \\
\hline
\end{tabular}

\section{Gas/particle}

Gas phase: The shear stress is related to the gradients of velocity components as:

$$
\sigma_{i j}=\mu_{g}\left[\left(\frac{\partial U_{j}}{\partial x_{i}}+\frac{\partial U_{i}}{\partial x_{j}}\right)-\frac{2}{3} \delta_{i, j} \frac{\partial U_{k}}{\partial x_{k}}\right]
$$

Where $\mu$ is the shear viscosity and $\delta_{\mathrm{ij}}$ is the Kroenecker delta. The turbulent viscosity in the gas phase is modelled with use of a Sub Grid Scale (SGS) model, first used by Deardorff (1971). The local Reynold's stresses, which arises from averaging over the finite difference cell are simulated by the SGS model. This SGS eddy coefficient is limited by the averaging domain, which in two dimensions is considered to be the grid volume $\Delta \mathrm{x} \Delta \mathrm{y}$.

The SGS model for turbulent viscosity can be written:

$$
\begin{gathered}
\mu_{g}=\rho_{g}\left(c_{i} \Delta\right)^{2} \sqrt{S_{i, j} \cdot S_{i, j}} \\
\text { with } c_{t}=0 \cdot 1 ; \Delta=\sqrt[3]{\Delta x \cdot \Delta y \cdot \Delta z} ; S_{i, j}=\frac{1}{2}\left(\frac{\partial U_{i}}{\partial x_{i}}+\frac{\partial U_{i}}{\partial x_{j}}\right)_{g}
\end{gathered}
$$

Solid phase: Here $\sigma$ is the stress tensor in the solid phase. The total shear stress in the solid phase is the sum of a collisional part and a kinetic part and may be formulated as:

$$
\sigma_{i, j}=-p_{s} \delta_{i j}+\xi_{s} \delta_{i j} \frac{\partial U_{k}}{\partial x_{k}}+\mu_{s}\left[\left(\frac{\partial U_{j}}{\partial x_{i}}+\frac{\partial U_{i}}{\partial x_{j}}\right)-\frac{2}{3} \delta_{i j} \frac{\partial U_{k}}{\partial x_{k}}\right]
$$

Where $\xi$ is the solid bulk viscosity. The solid phase pressure, $\mathrm{p}_{\mathrm{s}}$, which includes both kinetic and collisional pressures, is determined from an equation of state similar to the van der Waals equation of state for gases Chapman and Cowling (1970).

$$
p_{s}=\alpha_{s} \rho_{s}\left[1+2(1+e) \alpha_{s} g_{0}\right] \Theta
$$


Here, $e$ is the coefficient of restitution and $\mathrm{g}_{0}$ is the radial distribution function [Lun et al. (1984)] which is equal to one when the particles are loosely packed and becomes infinite when they are so closely packed that motion is impossible. $\Theta$ is the granular temperature related to the kinetic turbulent energy of the particle motion. A form of the radial distribution function adopted by Ding and Gidaspow (1990) is:

$$
g_{0}=\frac{3}{5}\left[1-\left(\frac{\alpha_{s}}{\alpha_{s, \max }}\right)^{\frac{1}{3}}\right]^{-1}
$$

Where, $\alpha_{s, \max }$ is the maximum solid volume fraction of a random packing. In the following computations, $\alpha_{s, \max }$ equal to 0.65 is used.

Solid phase bulk viscosity due to particle collisions can be written as:

$$
\xi_{s}=\frac{4}{3} \alpha_{s}^{2} \rho_{s} d_{p} g_{0}(1+e) \sqrt{\frac{\Theta}{\pi}}
$$

Here $d_{p}$ is the particle diameter.

The solid phase shear viscosity for dense and dilute flow is given by:

$$
\begin{gathered}
\mu_{s}=\frac{2 \mu_{s, d i l}}{(1+e) g_{0}}\left[1+\frac{4}{5}(1+e) g_{0} \alpha_{s}\right]^{2}+\frac{4}{5} \alpha_{s}^{2} \rho_{s} d_{p} g_{0}(1+e) \sqrt{\frac{\Theta}{\pi}} \\
\mu_{s, \text { dil }}=\frac{5}{96} \rho_{s} d_{p} \sqrt{\pi \Theta}
\end{gathered}
$$

The viscosity is seen to be a product of the mean free path times an oscillation velocity times a density.

The granular temperature or turbulent kinetic energy is determined by solving the following equation:

$$
\frac{3}{2}\left[\frac{\partial}{\partial t}(\alpha \rho \Theta)_{s}+\frac{\partial}{\partial x_{i}}\left(\alpha \rho U_{i} \Theta\right)_{s}\right]=\frac{\partial}{\partial x_{i}}\left[\Gamma_{\Theta} \frac{\partial \Theta}{\partial x_{i}}\right]+\sigma_{i j} \frac{\partial U_{j, s}}{\partial x_{i}}-3 \cdot \beta \cdot \Theta-\gamma
$$

Here $\Gamma$ is the transport coefficient or conductivity and $\gamma$ is the collisional energy dissipation expressed as:

$$
\gamma=3\left(1-e^{2}\right) \alpha_{s}^{2} \rho_{s} g_{0} \Theta\left[\frac{4}{d_{p}} \sqrt{\frac{\Theta}{\pi}}-\frac{\partial U_{k, s}}{\partial x_{k}}\right]
$$

For a restitution coefficient of one, that is perfectly elastic collision, no energy is lost in the collision and $\gamma$ equals zero.

Conductivity of fluctuation energy is:

$$
\begin{gathered}
\Gamma_{\Theta}=\frac{2 \Gamma_{\Theta, d i l}}{(1+e) g_{0}}\left[1+\frac{6}{5}(1+e) g_{0} \alpha_{s}\right]^{2}+2 \alpha_{s}^{2} \rho_{s} d_{p} g_{0}(1+e) \sqrt{\frac{\Theta}{\pi}} \\
\Gamma_{\Theta, d i l}=\frac{75}{384} \rho_{s} d_{p} \sqrt{\pi \Theta}
\end{gathered}
$$

\subsection{Interfacial friction}

Gas/liquid

A bubble moving in a liquid is subjected to several forces caused by the liquid flow. Some of the most important forces are the drag force or interfacial friction, resulting from the relative velocity between the two phases; the lift force caused by spin or velocity gradient of the surrounding liquid; and the added mass force resulting from 
an acceleration of the bubble. Modelling of gas-liquid interaction is further complicated by variation of bubble sizes caused by coalescence and breakup of the bubbles.

The formulation of the interfacial friction, which is considered the most important of the forces mentioned above, is approximate. Detailed formulation of the lift and added mass effect is therefore omitted at this stage. The gas and liquid momentum equations are then coupled by the interfacial friction terms, which in the $i^{\prime}$ th coordinate direction read

$$
F_{i, j}=\frac{3}{4} \alpha_{g} \alpha_{l} \rho_{l} \frac{C_{D}}{d_{b}}\left|\vec{V}_{g}-\vec{V}_{l}\right|\left(U_{i, g}-U_{i, l}\right)
$$

Amarasooriya and Theofanus (1988), who investigated premixing of steam explosions, applied friction coefficients dependent on the flow regimes; homogeneous bubble flow $\left(\alpha_{\mathrm{g}} \leq 0.3\right)$, heterogenous bubble flow $\left(0.3<\alpha_{\mathrm{g}} \leq 0.7\right)$ or droplet flow $\left(\alpha_{g}>0.7\right)$. The friction coefficients, which take into account the bubble-bubble interaction, were determined by Ishii and Zuber (1979). For bubbly flow $\left(\alpha_{g} \leq 0 \cdot 3\right)$ and droplet flow $\left(\alpha_{g}>0 \cdot 7\right)$, the friction coefficient is given by

$$
C_{D}=\frac{2}{3} d_{b} \sqrt{\frac{g \Delta \rho}{\gamma}}\left\{\frac{1+17.67\left[f\left(\alpha_{g}\right)\right]^{\frac{6}{7}}}{18.67 f\left(\alpha_{g}\right)}\right\}^{2}
$$

where $\quad \alpha_{\mathrm{g}} \leq 0.3: \quad \mathrm{f}\left(\alpha_{\mathrm{g}}\right)=\left(1+\alpha_{\mathrm{g}}\right)^{1.5}$

$$
\alpha_{\mathrm{g}}>0.7: \quad \mathrm{f}\left(\alpha_{\mathrm{g}}\right)=\alpha_{\mathrm{g}}{ }^{3}
$$

It can be noted that in these flow regimes, the interfacial friction term (equation 23) becomes independent of bubble size.

For churn turbulent flow $\left(0.3<\alpha_{\mathrm{g}} \leq 0 \cdot 7\right)$, the friction coefficient is given by

$$
C_{D}=\frac{8}{3} \alpha_{l}^{2}
$$

and

$$
d_{b}=\frac{4}{\sqrt{\frac{g \Delta \rho}{\gamma}}}
$$

where the surface tension, $\gamma$, takes the value $0.07\left[\mathrm{~kg} / \mathrm{s}^{2}\right]$ for water.

\section{Gas particle}

For the gas and solids momentum equations the interphase friction term read:

$$
\vec{F}_{k}= \pm \beta \cdot\left(\vec{V}_{s}-\vec{V}_{g}\right)
$$

Here $\beta_{\mathrm{ij}}$ is determined according to the following relations:

For $\alpha_{\mathrm{g}} \leq 0.8$ (based on the Ergun equation)

$$
\beta=150 \frac{\alpha_{s}^{2} \mu_{g}}{\alpha_{g}\left(d_{p} \phi_{s}\right)^{2}}+1.75 \frac{\alpha_{s} \rho_{g}\left|\vec{V}_{g}-\vec{V}_{s}\right|}{d_{p} \phi_{s}}
$$

For $\alpha_{\mathrm{g}}>0.8$ (based on empirical correlation)

$$
\beta=\frac{3}{4} C_{d} \frac{\left|\vec{V}_{g}-\vec{V}_{s}\right| \rho_{g} \alpha_{s}}{d_{p} \phi_{s}} \alpha_{g}^{-2.65}
$$


where

$$
\begin{array}{ccc}
C_{d}=\frac{24}{R e}\left(1+0.15 R e^{0.687}\right) \quad \text { for } & R e \leq 1000 \\
C_{d}=0.44 & \text { for } & R e>1000 \\
\operatorname{Re}=\frac{\left|\vec{V}_{g}-\vec{V}_{s}\right| \alpha_{g} \rho_{g} d_{p}}{\mu_{l, g}} &
\end{array}
$$

Here $C_{d}$ is the drag coefficient, Re is the Reynolds number and $\Phi_{s}$ is a measure of the sphericity.

\subsection{Interfacial mass transfer}

\section{Gas liquid}

The interphase mass transfer is taken account of through the source terms as follows:

$$
S_{m_{j, l}}=\left(k_{l} \cdot a\right)_{l} \cdot\left(C_{j, g}-C_{j, l}\right)
$$

The $\left(\mathrm{k}_{1} \mathrm{a}\right)$ is related to the dissipation rate, $\varepsilon_{1}$ gas fraction, $\alpha_{\mathrm{g}}$ and bubble diameter, $\mathrm{d}_{\mathrm{b}}$ (Trägårdh (1988)):

$$
\left(k_{l} a\right)=c_{m} \varepsilon_{l}^{0.2} \frac{6 \alpha_{g}}{d_{b}}
$$

\subsection{Interfacial heat transfer}

\section{Gas/particle}

Heat is generated by the catalytic exothermic solid phase chemical reaction. This heat is transported between the phases and cooled by generation of steam in the submerged heat exchanger.

Source term for the gas phase

$$
S_{h_{g}}=h_{v}\left(T_{p}-T_{g}\right)+h_{w}\left(T_{w}-T_{g}\right)
$$

Source term for the solid phase

$$
S_{h_{p}}=h_{v}\left(T_{g}-T_{p}\right)+h_{w}\left(T_{w}-T_{p}\right)+\sum_{k=1}^{k=n \text { reac }} \Delta H_{r x, k} \cdot r_{k}
$$

The effective transport coefficients are related to the turbulent viscosities as:

$$
\Gamma_{h, g}=\frac{\kappa_{\text {lam }, g}}{c_{p, g}}+\frac{\mu_{g}}{0.7} \quad \text { and } \quad \Gamma_{h, p}=\frac{\mu_{p}}{0.7}
$$

Where $\kappa_{\mathrm{lam}, \mathrm{g}}$ is the laminar conductivity and $\mathrm{c}_{\mathrm{p}, \mathrm{g}}$ is the specific heat capacity of the gas.

The volumetric heat transfer coefficient $h_{v}$ is calculated by using several different correlations for the Nusselt number $\mathrm{N}_{\mathrm{p}}$ in different flow regimes, characterized by the Reynolds number $\operatorname{Re}$ and the gas phase volume fraction $\alpha_{\mathrm{g}}$.

For $\alpha_{\mathrm{g}} \leq 0 \cdot 8$

$$
\begin{array}{lll}
N_{p}=(2+0 \cdot 106 \cdot R e) \cdot S_{p} & & R e \leq 200 \\
N_{p}=0 \cdot 123 \cdot\left(\frac{4 \cdot R e}{d_{p}}\right)^{0.83} \cdot S_{p}^{0.17} & & 200<R e \leq 2000 \\
N_{p}=0.61 \cdot R e^{0.67} \cdot S_{p} & & R e>2000
\end{array}
$$


For $\alpha_{g}>0 \cdot 8$

where

$$
\begin{aligned}
& N_{p}=\left(2+0 \cdot 16 \cdot R e^{0.67}\right) \cdot S_{p} \quad R e \leq 200 \\
& N_{p}=8.2 \cdot R e^{0.60} \cdot S_{p} \quad 200<\operatorname{Re}<1000 \\
& N_{p}=1.06 \cdot R e^{0.457} \cdot S_{p} \quad \operatorname{Re} \geq 1000
\end{aligned}
$$

$$
R e=\frac{\rho_{g} d_{p}\left|\vec{V}_{g}-\vec{V}_{p}\right|}{\mu_{\text {lam }, g}}, S_{p}=\frac{\alpha_{p} \cdot 6}{d_{p}}, N_{p}=\frac{h_{v} d_{p}}{\kappa_{\text {lam }, g}}
$$

The wall' to bed heat transfer coefficient, $h_{w}$, is for simplicity taken to be a constant, only proportional to the phase's respective volume fractions. A typical value for the bed to wall heat transfer coefficient used in this study is found in Geldart (1986).

\subsection{Stirred tank modifications}

The flow in the stirred tank may be calculated using a 2-D model description which neglects gradients in the tangential direction. The equations used for compuatations of agitated flow is mainly as given above, but additional equations and terms are introduced. The tangential velocity, $\mathrm{W}$, is calculated as a scalar variable as in equation 5. Additional terms appear on the right hand side of the momentum equations given by Hjertager and Morud (1993).

The Rushton impeller may, as a first approximation, be modelled as a rigid body motion with constant source term in the equation of tangential velocity. A more refined model is given by Pericleous and Patel (1987). This model introduces the influence of the Rushton impeller by an additional source term in the tangential momentum equation. The baffles are also modelled according to Pericleous and Patel (1987). Also in this situation an additional source is included in the tangential momentum equation.

\subsection{Kinetic modelling}

\section{Bioreaction}

A microbial kinetic model describing the reaction rates of the fed-batch fermentation of Saccharomyces cerevisiae is proposed by Enfors et al. (1992). The biomass growth rate is assumed to be the Monod type and include ethanol formation. Mass balances are made over each component in the liquid phase using the reaction rates given in Table 2.

The microbial kinetic model is based on the assumption that oxygen limitations are prevented for bakers' yeast processes operated as aerobic fed-batch fermenters. Thus the dissolved oxygen does not influence the reaction rate of any species. Consequently, the oxygen transport is not calculated and the conservation equation of oxygen is not listed above.

\section{Catalytic reaction}

Ozone decomposition reaction: The chemical reaction may be described as a simple homogeneous one step first order catalytic reaction and can be written as:

$$
2 \cdot \mathrm{O}_{3} \rightarrow 3 \cdot \mathrm{O}_{2}
$$

With the very low concentrations of ozone involved in the experiments of Ouyang

\footnotetext{
${ }^{\mathrm{I}}$ The word wall designates heat exchange surfaces immersed in a fluidized bed.
} 
Table 2. Source terms of chemical species.

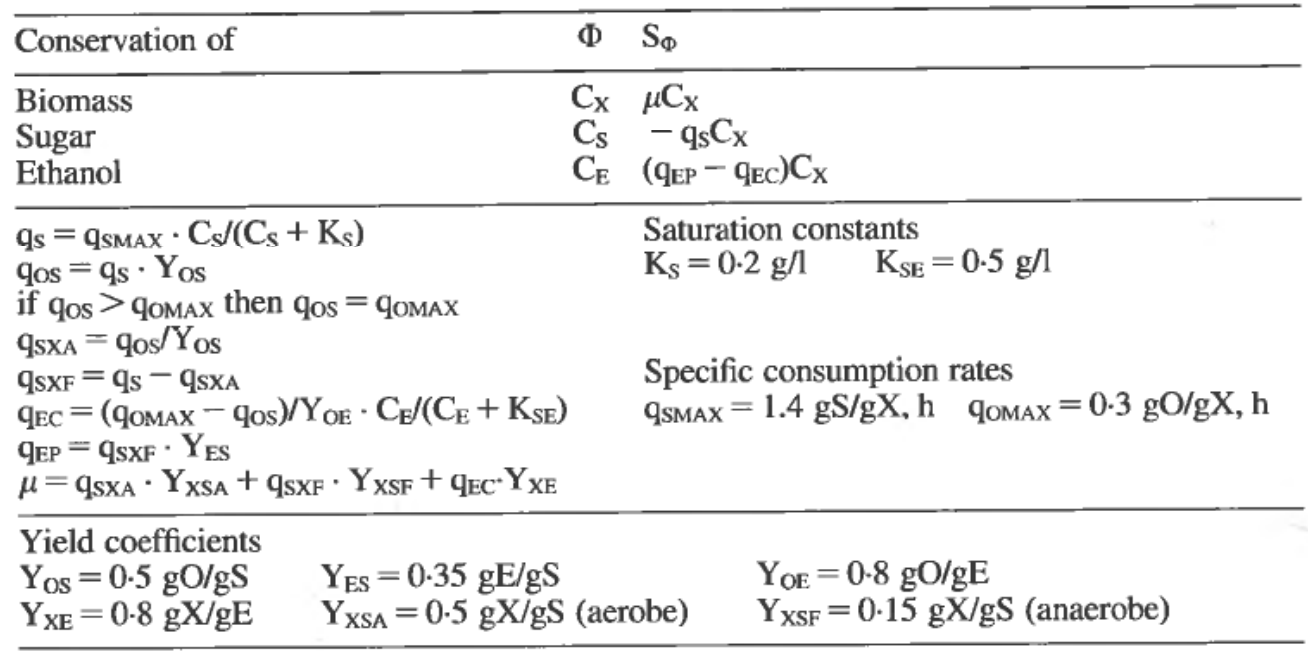

et al. (1993a, 1993b), the reaction heat produced during the reaction is negligible and the reaction can be looked upon as isothermal. The first order catalytic reaction decomposition rate constant based on the unit volume of the particles was measured to be equal to $3.96[1 / \mathrm{s}]$.

The reaction rate can then be written:

$$
S_{\text {Ozon }}=-3 \cdot 96 \cdot \alpha_{p} \cdot \rho_{p} \cdot Y_{\text {Ozon }} \quad\left[\frac{\mathrm{kg} \text { Ozon }}{\mathrm{m}^{3} \cdot \mathrm{s}}\right]
$$

Oxychlorination: Energy equations for the two phases are solved together with equations for the chemical species. The catalytic reactions take place on the surface of the catalyst. The simple one step homogeneous catalytic oxy-chlorination reaction can be represented by the following equation:

$$
2 \cdot \mathrm{C}_{2} \mathrm{H}_{4}+4 \cdot \mathrm{HCl}+\mathrm{O}_{2} \rightarrow 2 \cdot \mathrm{C}_{2} \mathrm{H}_{4} \mathrm{Cl}_{2}+2 \cdot \mathrm{H}_{2} \mathrm{O},-\Delta \mathrm{H}_{r x}=-240\left[\frac{\mathrm{kJ}}{\mathrm{mol}}\right]
$$

The rate of reaction is given as a function of the partial pressure of ethen as:

$$
r=k \cdot p_{C_{2} H_{4}} \quad\left[\frac{m o l E D C}{h \cdot g_{c a t}}\right]
$$

where the specific reaction rate is given by the usual Arrhenius equation:

$$
k=k_{1} \cdot e^{-\left(\frac{E}{R \cdot T}\right)}
$$

To convert the component's partial pressures into massfractions $\left(Y_{j}\right)$, the ideal gas law is used. The rate of reaction in equation 5 can then be written as:

$$
S_{Y_{E D C}}=k \cdot \alpha_{p} \cdot \rho_{p} \cdot M_{E D C} \cdot Y_{C_{2} H_{4}} \cdot \frac{R_{i}}{R} \cdot p_{t o t} \quad\left[\frac{k g E D C}{s \cdot m^{3}}\right]
$$

\section{Numerical procedure}

The partial differential equations are expressed by algebraic so called "finite domain" equations by dividing the calculation domain into several control volumes and by integration over these control volumes for each phase with respect to time and space. The discretizations are done by using interpolation functions, here the upwind scheme described in the book of Patnaker (1980) is used for space, and a fully implicit scheme 
for time. The momentum equations are coupled through the drag terms and when solving the equations sequentially, the convergence may be slow for large drag terms. This is avoided by using the partial elimination algorithm (PEA). Point iteration has been used to solve the volume fraction and momentum equations. The equations are solved sequentially, and because of the nonlinearities and coupling between the equations there is a need for an iterative solution procedure. The single phase solution algorithm SIMPLE (Semi-Implicit Method for Pressure Linked Equations) by Patankar and Spalding (1972) extended for two phase flow by Spalding (1985) has been used.

After a converged solution of the gas/liquid flow is obtained, the flow variables $(\alpha$, $\mathrm{U}, \mathrm{V}, \mathrm{r} \cdot \mathrm{W}, \mathrm{k}, \varepsilon$ and $\mathrm{p}$ ) are fixed, and only the equations governing the biochemical species are solved. This is to enable different time steps for the simulation of flow and biochemical reactions. The time steps for the flow simulations are calculated from a specified Courant number. The bioconversions to be simulated last for typically ten hours, and the time steps were chosen based on a sensitivity test of the actual process.

\section{Prediction of gas/liquid flow processes in stirred vessels}

Simulations of flow and biochemical conversion in small, medium and large scale stirred vessels have been performed (Morud, 1994) using the model for gas/liquid flows given above. The simulations of the small scale vessel are verified against the LDA/PDA results presented by Morud and Hjertager (1996). The numerical results of the medium and large scale vessels are compared against experimental data Norman et al. (1993a, b, c).
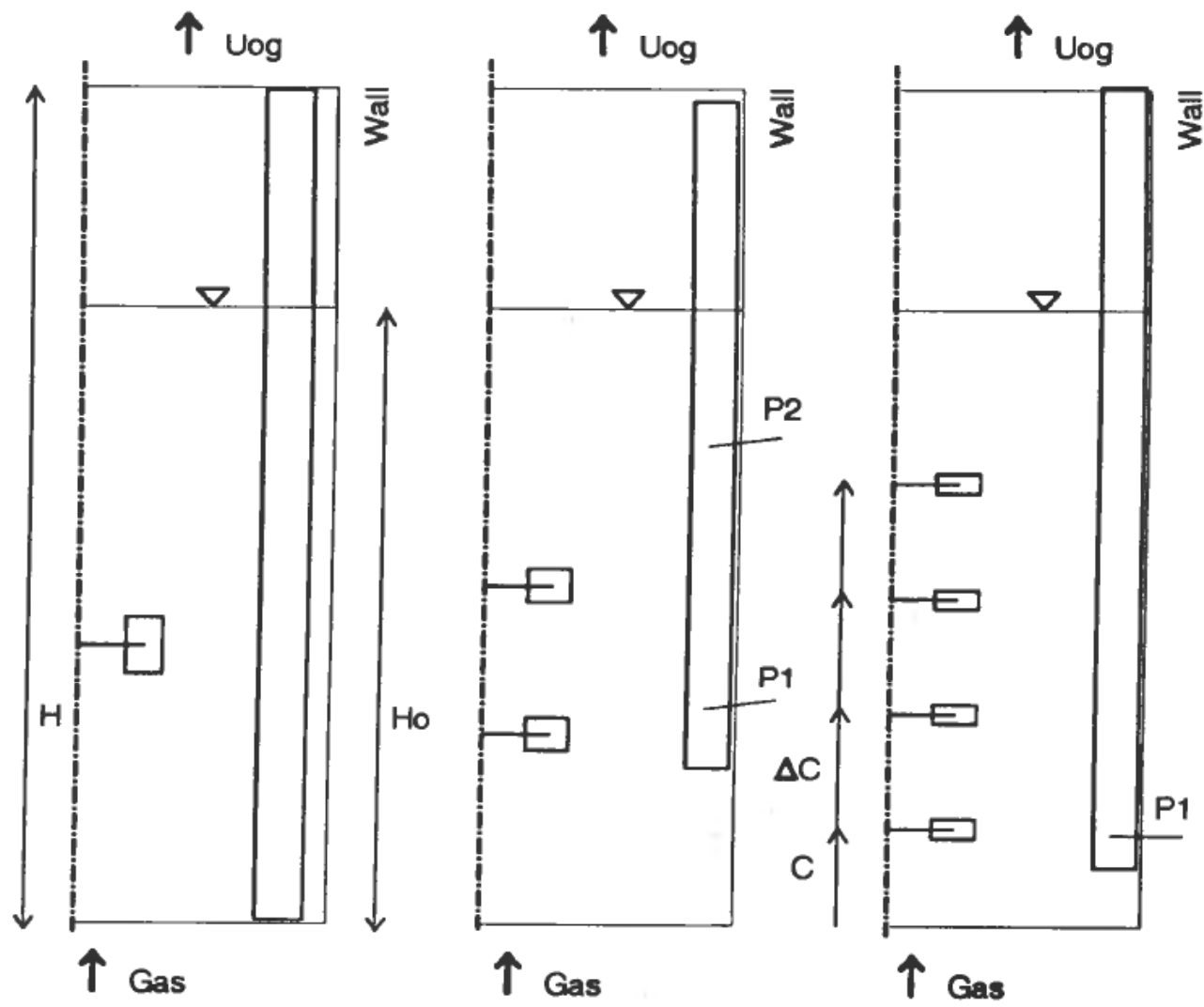

Figure 2. Stirred vessel configurations.

a) vessel 1, b) vessel II and c) vessel III. 


\subsection{Configurations}

Figure 2 shows the configurations of the small, medium and large scale stirred vessels, denoted vessel I, II and III, respectively. The corresponding vessel dimensions are given in Table 3. The vessel configurations are noted to be axisymmetric. All vessels are modelled with flat bottoms and with gas inlets through the bottoms below the impellers. An area porosity, $\beta_{\mathrm{x}}$, is used at the inlet, determining the fraction of inlet area for flow. Vessel I represents the 15 litre vessel described by Morud and Hjertager (1996). Vessel II represents a $1 \mathrm{~m}^{3}$ vessel used in the experiments of Noorman et al. (1993b). A bakers' yeast process of vessel III [Noorman et al., 1993a] is simulated with sugar feed at the vessel wall. The locations of the measurement ports (denoted P1 and P2) are shown in the Figure.

Table 3. Vessel dimensions.

\begin{tabular}{lccc}
\hline & Vessel I & Vessel II & Vessel III \\
\hline Vessel diameter, D[m] & 0.222 & $0 \cdot 90$ & 2.09 \\
Impeller diameter, $\mathrm{D}_{\mathrm{i}}$ & $\mathrm{D} / 3$ & $\mathrm{D} / 3$ & $\mathrm{D} / 3$ \\
Diameter gas inlet & $\mathrm{D} / 5 \cdot 5$ & $\mathrm{D} / 6$ & $\mathrm{D} / 4$ \\
Ungassed liquid level, $\mathrm{H}_{0}$ & $1.0 \mathrm{D}$ & $1.10 \mathrm{D}$ & $2.85 \mathrm{D}$ \\
Height, H & $1.35 \mathrm{D}$ & $1.67 \mathrm{D}$ & $4.42 \mathrm{D}$ \\
No. of impellers & 1 & 2 & 4 \\
No. of baffles & 4 & 3 & 4 \\
Impeller clearance, C & $0.47 \mathrm{D}$ & $0.33 \mathrm{D}$ & $0.47 \mathrm{D}$ \\
Impeller spacing, $\Delta \mathrm{C}$ & - & $0.30 \mathrm{D}$ & $0.62 \mathrm{D}$ \\
\hline
\end{tabular}

The operating conditions for the vessels are given in Table 4. For the three vessels, the grid resolutions are chosen so that each impeller is described with two control volumes in the axial direction, resulting in the following three grids: $47 \times 24,52 \times 26$ and $140 \times 26$.

Table 4. Operating conditions.

\begin{tabular}{|c|c|c|c|c|c|c|}
\hline \multirow[b]{2}{*}{$\begin{array}{l}\mathrm{N}[\mathrm{RPM}] \\
\mathrm{W}_{\text {tip }}[\mathrm{m} / \mathrm{s}] \\
\mathrm{Q}[\mathrm{VVM}]\end{array}$} & \multicolumn{3}{|c|}{ Vessel I } & \multicolumn{2}{|c|}{ Vessel II } & $\begin{array}{c}\text { Vessel III } \\
133\end{array}$ \\
\hline & $\begin{array}{l}360 \\
1 \cdot 4 \\
0.49\end{array}$ & $\begin{array}{l}540 \\
2.1 \\
1.00\end{array}$ & $\begin{array}{l}720 \\
2 \cdot 8 \\
1 \cdot 33\end{array}$ & $\begin{array}{r}300 \\
4.7 \\
0 \cdot 0\end{array}$ & 0.7 & $\begin{array}{c}133 \\
4.9 \\
0.54\end{array}$ \\
\hline $\begin{array}{l}\beta_{\mathrm{x}} \text {, gas inlet }[-] \\
\text { media }\end{array}$ & $\begin{array}{c}0.01 \\
\text { non-c } \\
\text { coales }\end{array}$ & scing & & $\begin{array}{r}0 \cdot 01 \\
\text { non-c } \\
\text { coale }\end{array}$ & scing & $\begin{array}{c}0.02 \\
\text { coalescing }\end{array}$ \\
\hline
\end{tabular}

In the presentation of the numerical results, the velocities are given in $[\mathrm{m} / \mathrm{s}]$ and the radial positions are normalized by the reactor radius i.e. $r^{*}=r / R$.

\subsection{Flow pattern}

Gas flow pattern

Figure 3 shows typical gas flow patterns from the simulation of vessel I, II, and III. In vessel I, gas is seen to rise up to the impeller and then move radially towards the wall. Two circulation zones are formed. A large one is formed just above the impeller, 

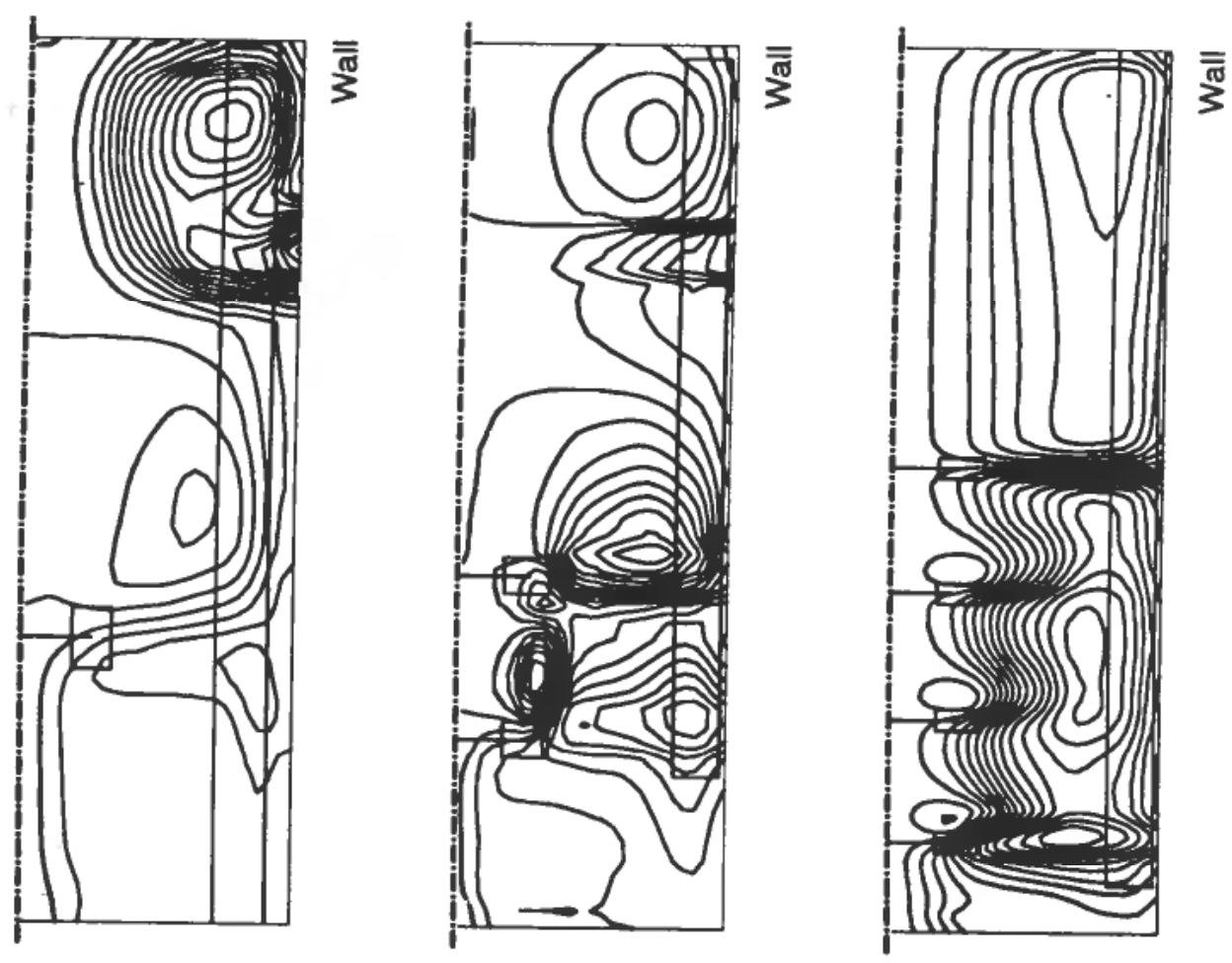

Figure 3. Predicted streamlines gas.

a) vessel I, b) vessel II and c) vessel III.

whereas a smaller one is formed just below the impeller. The Figure also demonstrates the gas circulation above the liquid surface. Vessel II, which is equipped with two narrowly spaced impellers, produces a flow pattern quite similar to that of the small scale vessel. However, a third recirculation zone is observed to appear between the two impellers. The gas flow in vessel III follows a characteristic zigzag pattern with a small recirculation zone above each impeller. A similar flow pattern has been observed by Bröring et al. (1991), measuring gas velocities with the Ultrasound-Pulsed-Doppler technique in a vessel equipped with 3 Rushton turbines.

\section{Liquid flow pattern}

Vessel II is simulated with and without gas sparger. Figure 4 indicates how the liquid flow pattern is influenced by the gas flow. For the gas flow rate $Q=0.7 \mathrm{VVM}$, an outflow from the upper impeller is predicted, whereas the outflow is located midway between the impellers when the vessel is ungassed. Hudcova et al. (1989) and Mahmoudi and Yianneskis (1991) have studied the effect of impeller spacing on liquid flow. The predicted outflow midway between the two impellers of vessel II, is in good agreement with their observations of single phase flow for narrow impeller spacing $\left(\Delta \mathrm{C} \leq \mathrm{D}_{\mathrm{i}}\right)$.

\subsection{Gas fraction}

Contour plots of the simulated gas fraction are shown in Figure 5. Generally, regions with high gas fraction are noticed to coincide with the regions of recirculated gas 

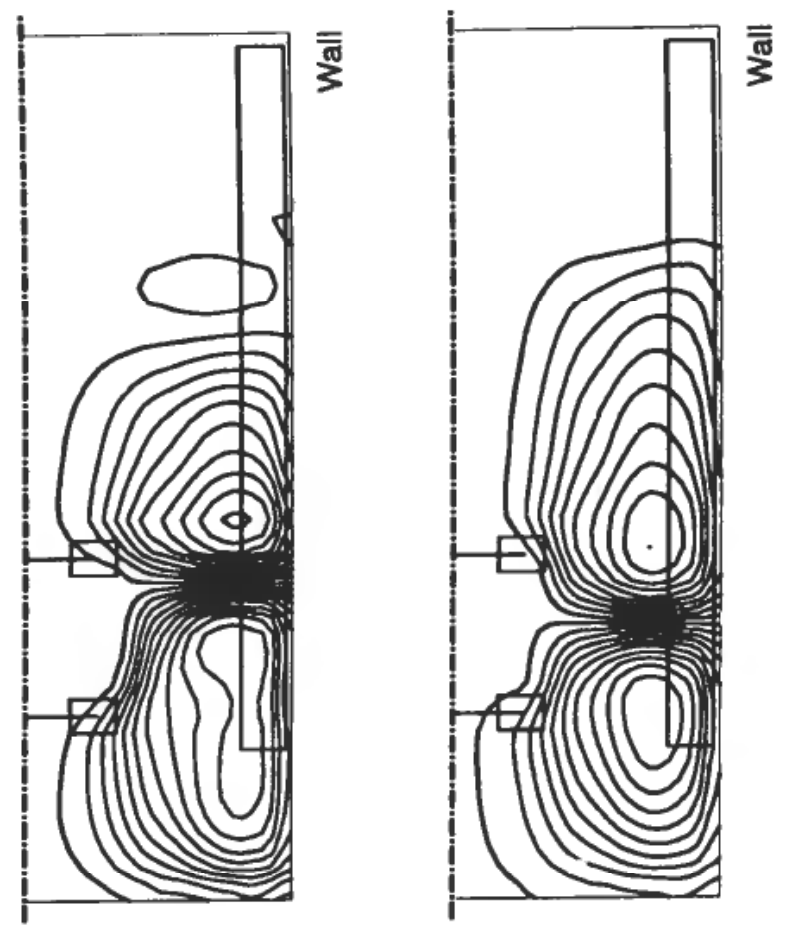

Figure 4. Predicted streamlines liquid of vessel II.

a) with gas flow $Q=0.7 \mathrm{VVM}$ and $\mathrm{b}$ ) ungassed, $\mathrm{Q}=0.0 \mathrm{VVM}$.
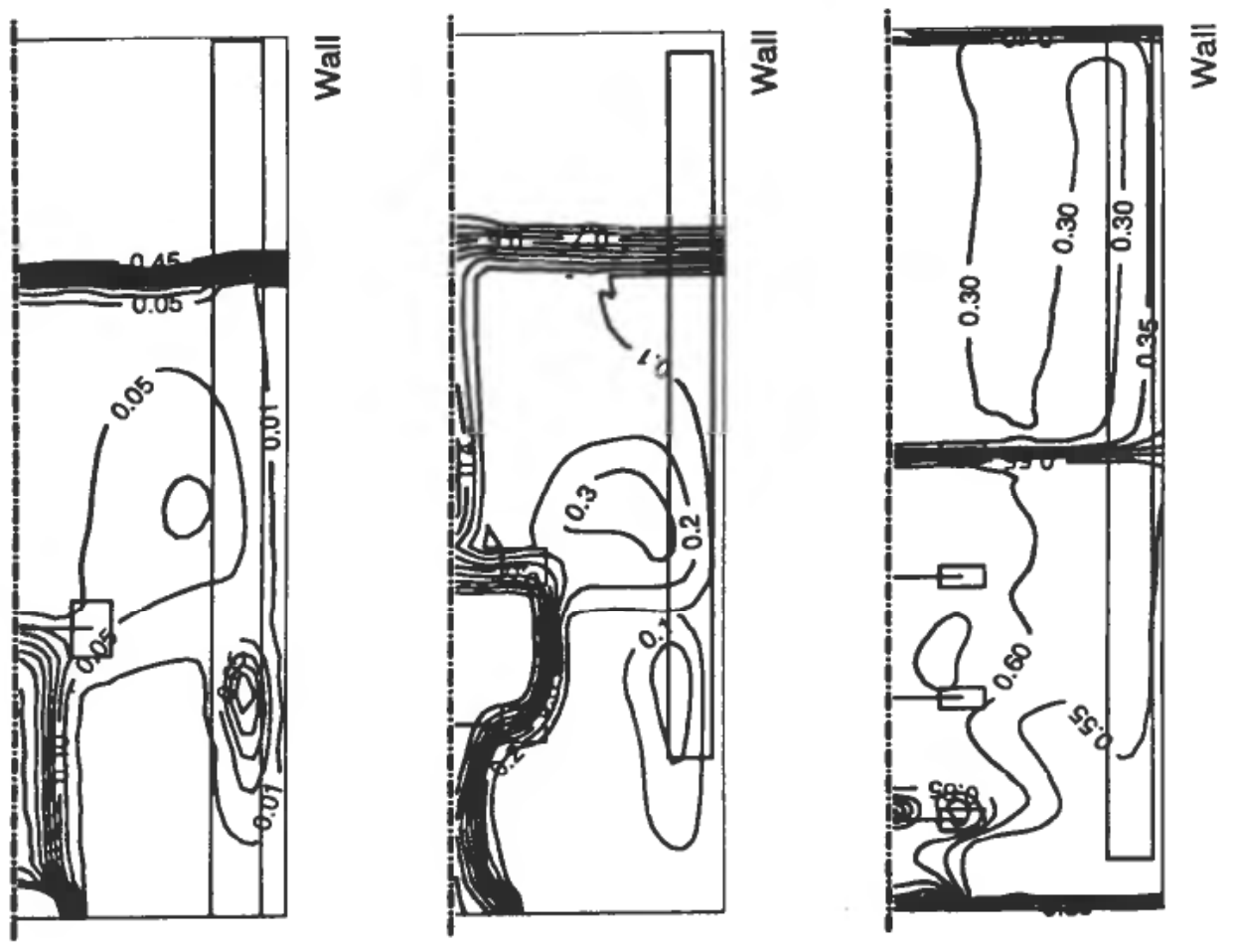

Figure 5. Predicted gas fraction contours.

a) vessel I $(\mathrm{Q}=1.0 \mathrm{VVM})$, b) vessel II $(\mathrm{Q}=0.7 \mathrm{VVM})$ and $\mathrm{c})$ vessel III $(\mathrm{Q}=0.54 \mathrm{VVM})$. 
flow shown in Figure 3. For vessel I, the gas fraction is high between the gas inlet and the impeller, in the upper part of the vessel and in a smaller zone near the baffles. In the lower part of the vessel, there is a large zone with a very low gas fraction which is also observed experimentally.

The gas fraction contours of vessel II show larger gas fractions, but a fairly similar description to that of vessel I. Between the two impellers a gas pocket is formed, which appears to remain even if the gas is turned off in the simulations. According to the observations of Hudcova et al. (1989) and Mahmoudi and Yianneskis (1991), the liquid trapped between the two impellers has little evidence of any radial motion. Obviously, the swirling motion between the two impellers is very strong. In a 3-D formulation, the centrifugal force would vary in angular direction, and the gas most likely would rise. Strong centrifugal forces for $2 \mathrm{D}$ models means that the gas is forced towards the centre of the vessel in the whole periphery of the region between the two impeller tips. Hence, the gas will remain between the two impeller discs. Near the axis in the upper part of the vessel the gas and liquid are separated. This is caused by the highly swirling motion. An increase of gas fraction near the axis was also found experimentally by Bakker (1992) using an optical fibre probe in a stirred tank equipped with a Rushton turbine. Noorman et al. (1993b) measured total gas fraction in the vessel. In desalinated water and in mineral salt medium the measured fractions were 0.085 and 0.11 respectively, $\pm 1 \%$. The simulations give a total gas fraction of $0 \cdot 15$, where the gas pocket occupies approximately $1 \%$ of the total gas/liquid volume.

The predicted gas fraction contours of vessel III indicate that the fractions are very high below the upper impeller. As indicated in the Figure, the liquid surface has reached the top of the calculation domain. The simulations are performed with an initial liquid level of $2.85 \mathrm{D}$. Thus, the predicted total gas fraction is more than 0.35 . The measured total gas fraction in tap water and during fermentation were 0.17 and 0.14 respectively [Noorman et al., 1993a]. Clearly, the simulated gas fraction is far higher than the measured values. Several reasons may explain the large difference between the predicted and simulated total gas fractions. Calculations for a bubble column indicate that the predicted total gas fraction compares fairly well with the measured ones for water-ethanol solutions, but is almost twice as high as the measured ones for water. It is therefore reasonable to believe that the numerical results will improve by taking coalescence into account. Moreover, the hydrostatic pressure is not accounted for in the simulations, a feature that is assumed to have some effect for vessels of this size.

\section{Small scale vessel}

Total gas fraction The simulation results of vessel I are compared against the total gas fraction observations given by Morud and Hjertager (1996). In Figure 6, the computed and measured total gas fractions are plotted versus the gas flownumber $\mathrm{Fl}_{\mathrm{g}}=\mathrm{Q} /\left(\mathrm{ND}_{\mathrm{i}}{ }^{3}\right)$. The agreement between the predicted and measured total gas fraction is good, and the simulated fractions lie within the error range of the experimental data.

\subsection{Mean flow}

Small scale vessel

Radial velocities The predicted and measured radial gas velocities at one level in the vessel are given in Figure 7 for the two gas flow rates $\mathrm{Q}=0.49 \mathrm{VVM}$ and 1.0 VMM.

Above the impeller level, the gas is flowing with low velocity towards the centre axis. The predicted velocities show good agreement with the experimental data. At the 


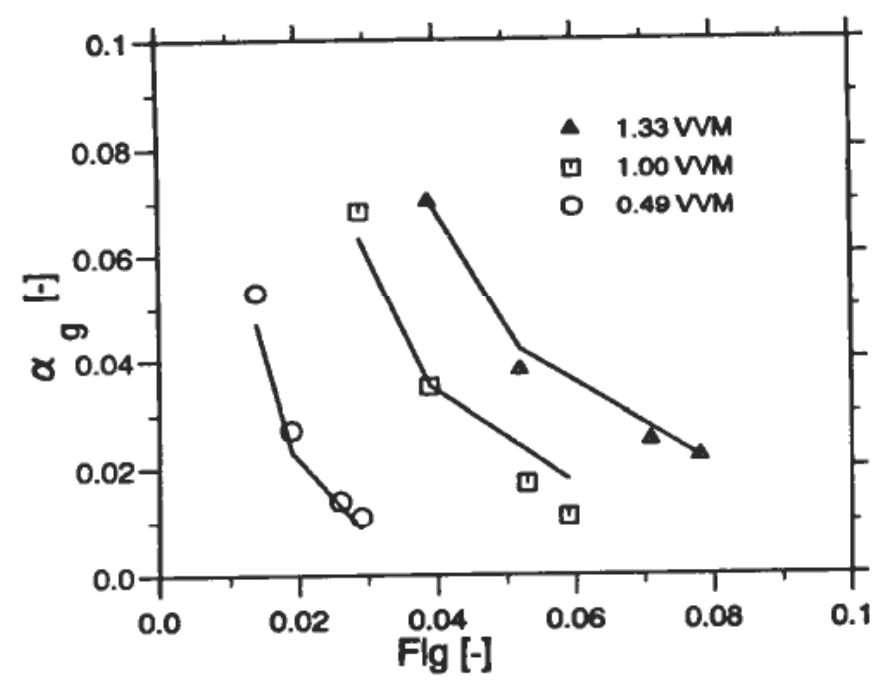

Figure 6. Simulated and measured total gas fraction to vessel I.
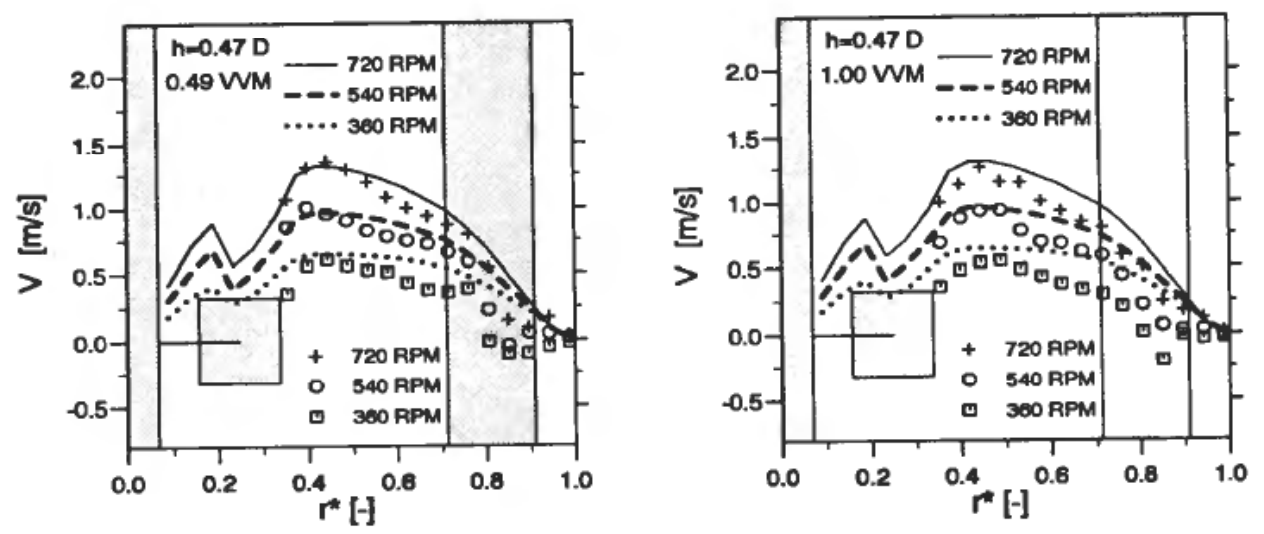

Figure 7. Simulated and measured radial gas velocity of vessel 1. a) $\mathrm{Q}=0.49 \mathrm{VVM}$ and b) $\mathrm{Q}=1.00 \mathrm{VVM}$.

level of the impeller, the predicted increase of radial gas velocity just outside the impeller tip agrees well with experimental data for all rotational speeds. The measured radial backflow that occurs for lower rotational speeds is, however, not captured in the simulations. Below the impeller, the radial gas velocity profiles are similar to the ones above the impeller. The simulated velocities fit well to the experimental data except in the centre of the vessel. Here, the measured velocities are higher than for the rest of the profile and the gas is pushed outwards, away from the gas sparger. As shown in the experimental setup in Morud and Hjertager (1996), the gas inlet is located midway between the impeller disc and the vessel bottom. A simplification is made in the simulations, i.e. the gas inlet is placed at the vessel bottom. The profile of simulated velocity presented is therefore further away from the gas inlet than for the experiments.

Figure 8 compares the predicted radial gas and liquid velocities at the plane of the impeller. In the impeller region, there is a strong swirl. The centrifugal force acts most 


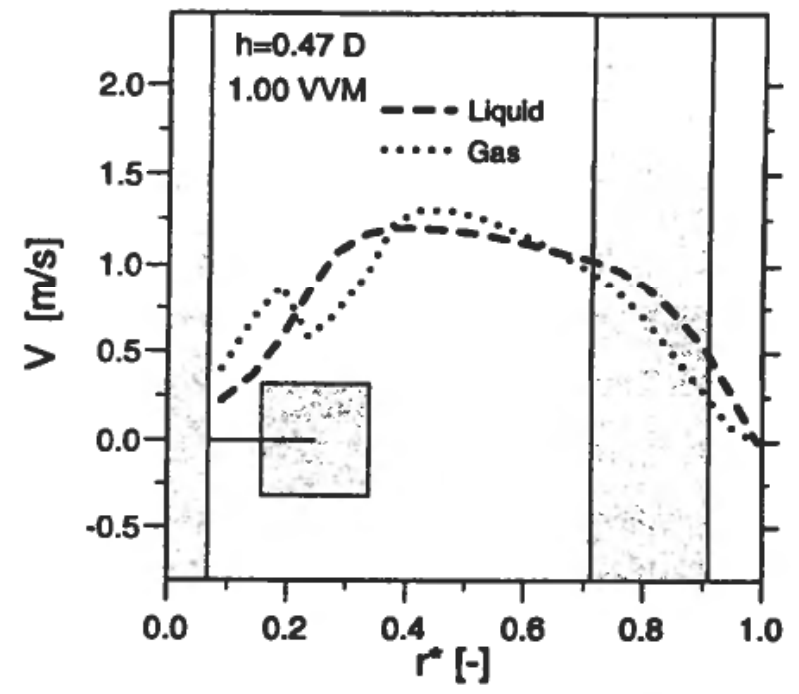

Figure 8. Simulated radial gas and liquid velocity of vessel I.

dominantly on the heaviest phase, tending to separate the gas and liquid phase. Consistently, the liquid velocity is noted to be higher than the gas velocity at the impeller tip. The liquid velocity decreases monotonically towards the wall, whereas the gas, which is accelerated by the liquid, reaches its maximum velocity at about $r^{*}=0.41$.

Axial velocities Figure 9 shows profiles of the axial gas velocity at one height in the vessel. Above the impeller, the predictions fit the experimental data quite good in the central part of the vessel $(r / R \leq 0 \cdot 6)$. Closer to the wall, the measurements are overpredicted, a feature that is caused by the assumptions of axisymmetric flow in the calculations. This assumption is, however, violated by the presence of baffles. In analyzing the data, attention is therefore focused on the flow close to the impeller and

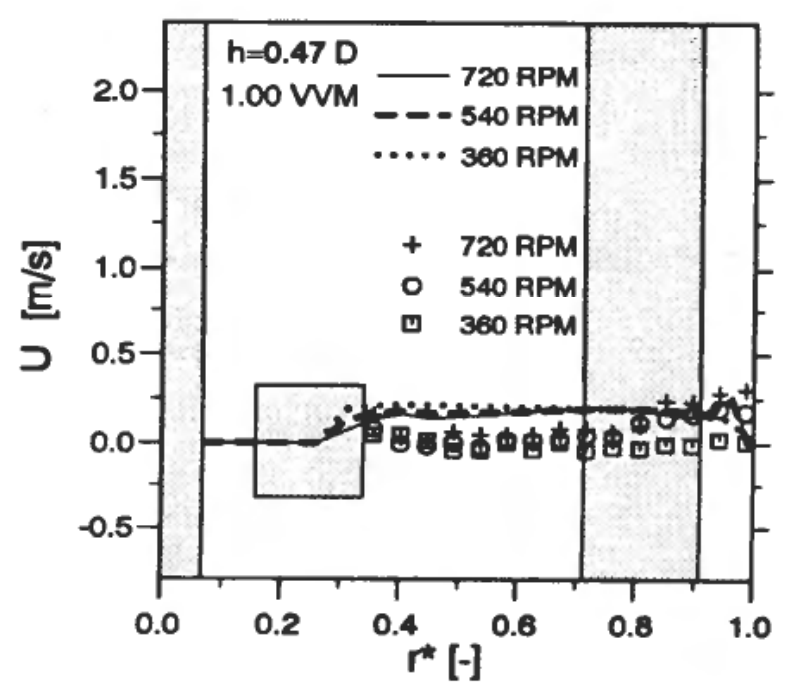

Figure 9. Simulated and measured axial velocity of vessel I. 
shaft rather than the flow near the baffle. At the height of the impeller, the simulations show that gas rises, whereas the measured data also show some negative velocities. The simulations show that with increasing impeller speed the axial gas velocity is reduced. Below the impeller, profiles of both predicted and measured data have a steep slope close to the shaft, particularly for the lower impeller speed. The simulations and measurements indicate the same flow direction, but the simulations overpredict the measured axial gas velocity. From the upper and lower level it is observed how the absolute value of the predicted axial velocity increases with increasing impeller rotational speed.

Tangential velocities Figure 10 shows the simulated and measured tangential gas velocities at one level. Above the impeller, the tangential gas velocity is low and the predictions seem to follow the experimental data fairly well. The tangential gas velocity at the impeller height is high and at the first few points near the impeller tip there is good agreement between the experimental data and the simulated profile.

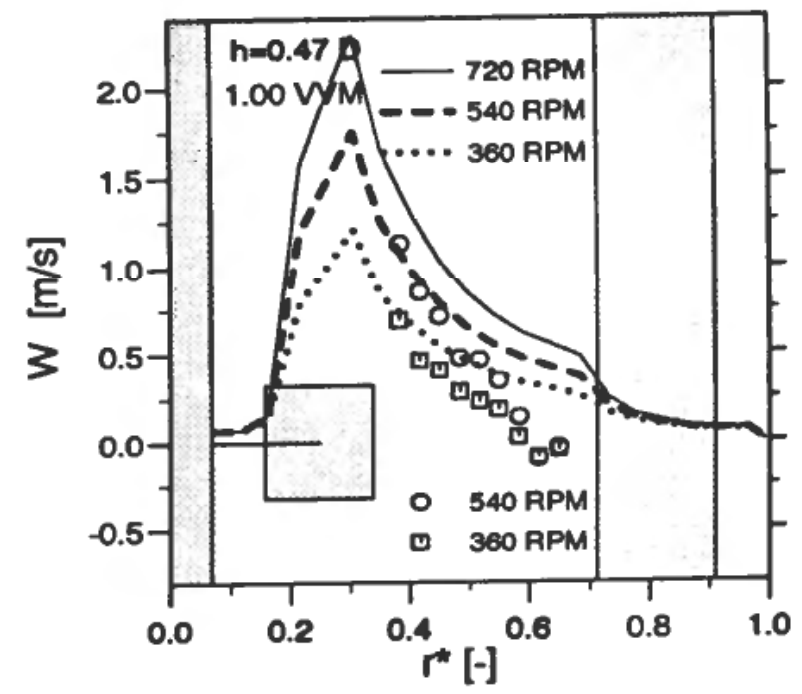

Figure 10. Simulated and measured tangential gas velocity of vessel I.

\section{Medium scale vessel}

Radial velocities A comparison between predicted and measured profiles of radial liquid velocities at the height of the lower impeller of vessel II is shown in Figure 11. The experimental data were obtained with a propeller anemometer by Noorman $\mathrm{et}$ al. (1993b), for a gas flow rate $Q=0.7 \mathrm{VVM}$ in water. The deviation between the simulated and the measured values is noted to be significant. In most of the profile, the simulated velocities are negative, while the experimental data are all above $0.2 \mathrm{~m} / \mathrm{s}$. As the name indicates, the propeller anemometer consists of a propeller that is set in motion and rotates with the velocity of the fluid flow. The propeller measurements are sensitive to being placed normal to the flow direction, and are not able to reveal the occurrence of flow reversal. The measurements were performed on the assumption that at the impeller height, the dominating velocity component would be in the radial direction. When aerated, the flow is hard to visualize. However, single phase flow visualization performed by Hudcova et al. (1989) and Mahmoudi and Yianneskis (1991) for narrowly 

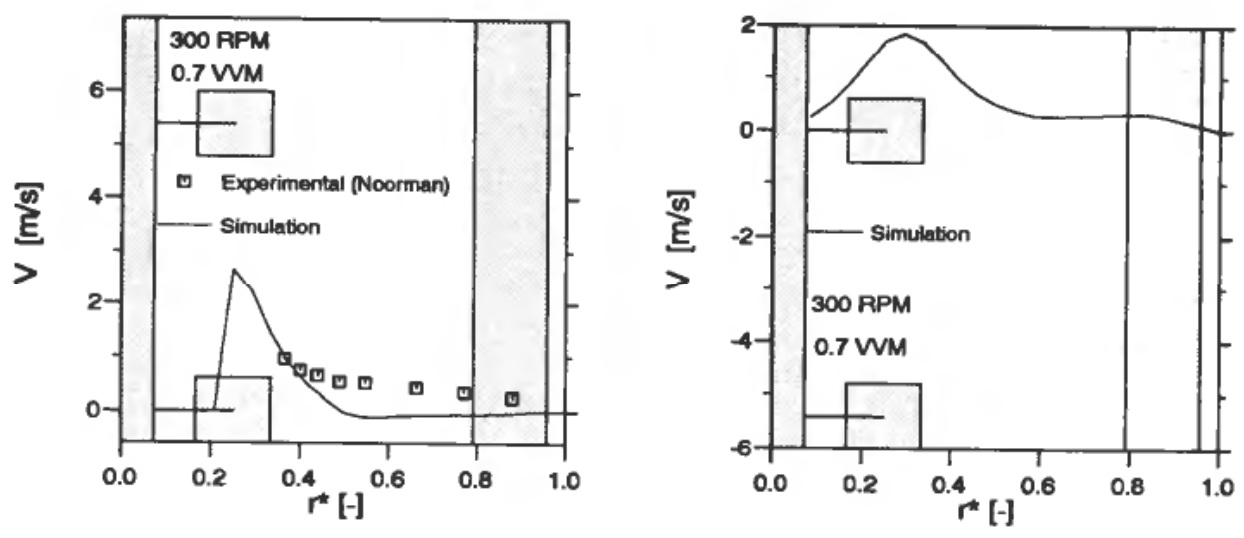

Figure 11. Comparison between present predicted radial liquid velocity of vessel II (lines) and measurements of Noorman et al. (1993b) (markers). (a) lower impeller and (b) upper impeller.

spaced impellers did not confirm the above assumption. Gas bubbles are most likely affecting the propeller measurements. It is also possible that the simulated radial velocity in the impeller region is reduced by the large gas pocket between the impellers.

\section{Large scale vessel}

Radial velocities In Figure 12 profiles of predicted radial liquid velocity are compared against propeller measurements of Noorman et al. (1993a). The profiles are taken at the level of the first and third impeller from below, at a gas flow rate of $Q=0.54 \mathrm{VVM}$ in tap water. Deviations between predicted and measured radial velocities are found. The predicted values are much lower than the experimental data at both heights. The impeller spacing for this reactor is larger than one impeller diameter, and the assumption of non-interacting impellers, with radial outflow from each impeller, is therefore more likely than for vessel II. In certain cases zizgag flow pattern has been found [Bröring et al., 1991]. The large deviations between the predicted and experimental results must be seen in relation to the overestimated total gas fraction discussed above.
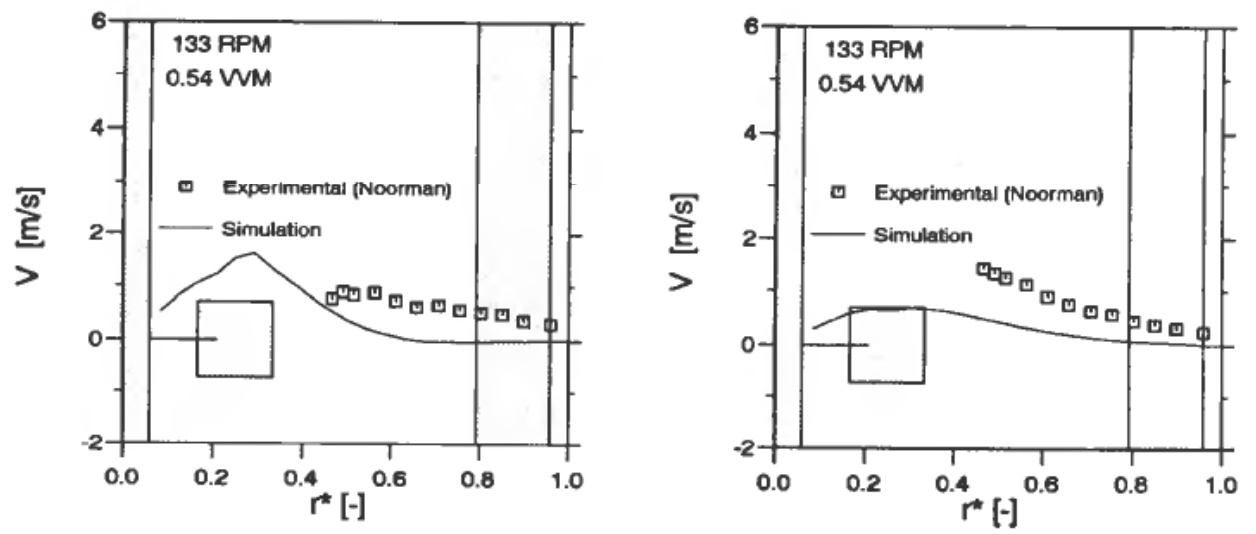

Figure 12. Comparison between present predicted radial liquid velocity of vessel III (lines) and measurements of Noorman et al. (1993a). (a) lower impeller and (b) third impeller from below. 

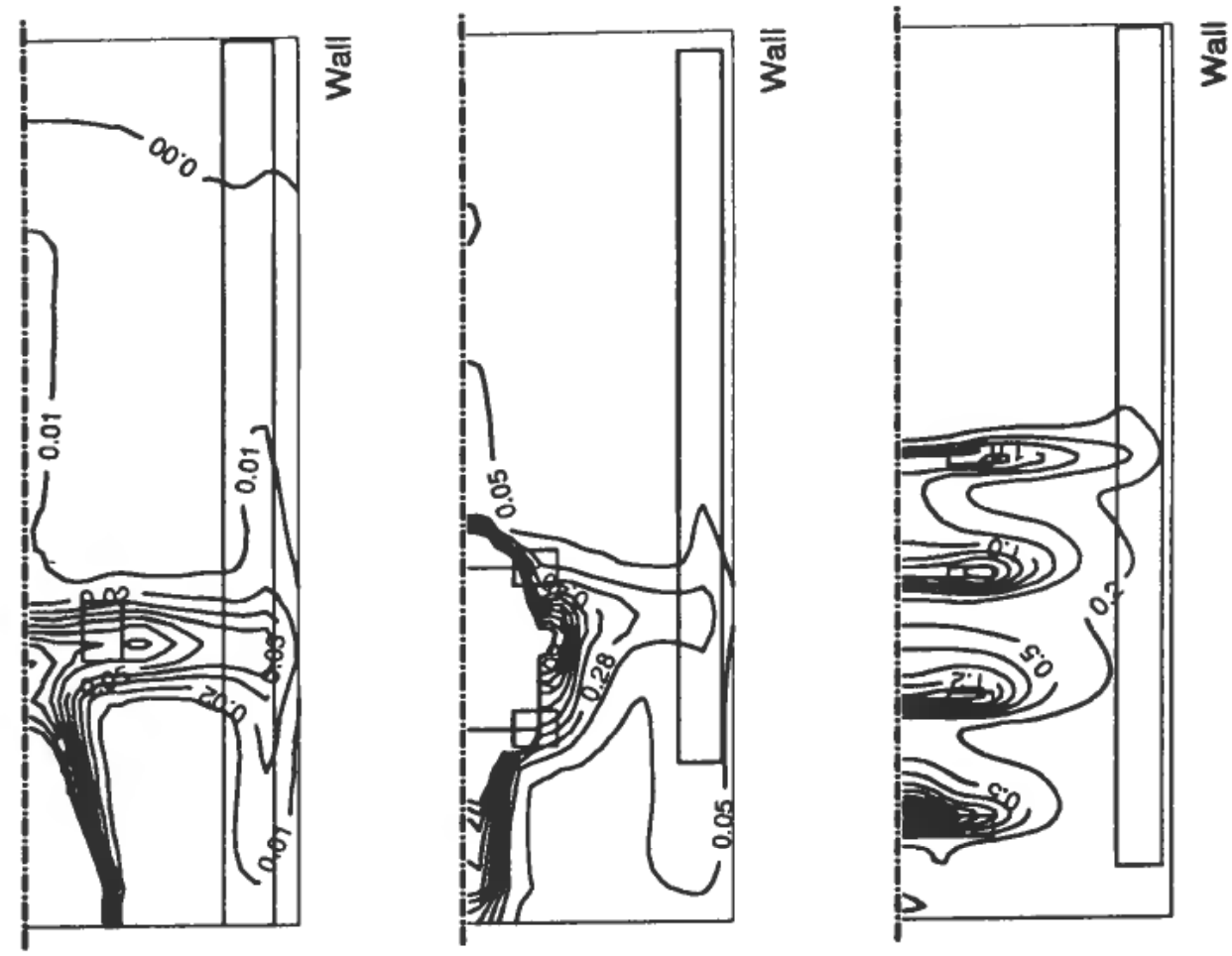

Figure 13. Predicted contours of turbulent kinetic energy.

a) vessel I, b) vessel II and c) vessel III.

\subsection{Turbulence}

Contours of turbulent kinetic energy for the three vessels are shown in Figure 13. High turbulent kinetic energies are found near the gas inlets and in the impeller regions. Integration of the predicted local turbulence dissipation rates of the medium scale vessel (vessel II), gives an impeller power input of $\mathrm{P}_{\mathrm{g}} / \mathrm{V}_{1}=1.3\left[\mathrm{~kW} / \mathrm{m}^{3}\right]$. The calculation of the impeller power input using empirical correlations gives a much higher result; $\mathrm{P}_{\mathrm{g}} / \mathrm{V}_{1}=2 \cdot 2\left[\mathrm{~kW} / \mathrm{m}^{3}\right]$ [Noorman et al., 1993b]. Used in the empirical correlations are power numbers of $4 \cdot 4$ and $3 \cdot 9$, determined by Hudcova et al. (1989) for narrowly spaced impellers. For the large scale vessel, impeller power input determined by simulations and by measurements [Noorman et al. (1993a)] are $330\left[\mathrm{~W} / \mathrm{m}^{3}\right]$ and $760\left[\mathrm{~W} / \mathrm{m}^{3}\right]$, respectively. For both medium and large scale vessels, the simulated impeller power input is observed to be much lower than that determined by correlations. Accordingly, further work on verifications of local turbulence quantities is needed. Here, the single phase $\mathrm{k}-\varepsilon$ model is assumed to be applicable to the continuous phase, but other models may be considered. Recently, Lahey (1992) presented a turbulence model for two phase flow which seems to predict well upflow and downflow in pipes. The Reynolds stresses for the liquid phase were composed of a shear induced part determined by the k- $\varepsilon$ model and a bubble induced part.

\subsection{Mixing times and biochemical reaction}

\section{Medium scale vessel}

Tracer response A tracer response is simulated for the medium scale vessel (vessel II). The tracer is injected just below the liquid surface near the shaft, and the detection point 
is located near the wall, at the height of the lower impeller (port P1, Figure 2b)). Different timesteps are tested initially, and a timestep of $0.1 \mathrm{~s}$ is chosen. Experiments with a pH electrode with a first order time constant of $0.7 \mathrm{~s}^{-1}$ are performed by Noorman et al. (1993b). Experimentally an ideal pulse is not easily obtained. In addition to the response time of the electrode, the experimental response curve will to some extent be delayed also due to the injection time. No delay was introduced in the simulations. Figure 14 compares the simulated tracer response curve against four experimental parallels [Noorman et al., 1993b]. The Figure shows concentration normalized by the final concentration.

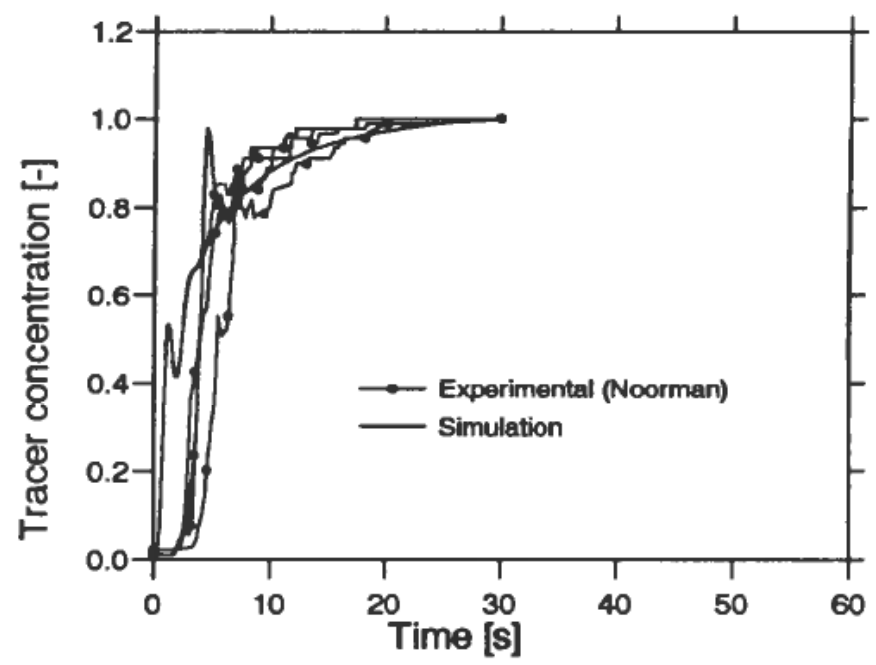

Figure 14. Predicted tracer response curves of vessel II against the measurements of Noorman et al. (1993b). Q = 1.0 VVM, N=300 RPM.

From the figure the time before complete mixing is seen to be well predicted. A $90 \%$ mixing time, defined as the time elapsed before the concentration is within $10 \%$ of the final value, is for the experiment parallels between 12 and 17 seconds and for the simulation 17 seconds. At the first part of the tracer response, simulation results shown an almost immediate response to the injected pulse, a feature that contradicts the experimental results. The simulations revealed that the response curve differed substantially when choosing detection points at other heights or radial positions, especially during the first seconds of the response time. The experimental curves will possibly change depending on the tangential positions of the detection points relative to the baffles. With these 2-D simulations it is therefore considered to be of most interest to compare the $90 \%$ mixing time (or similar) rather than focusing on the first part of the curve.

Bakers' yeast process The distribution of sugar of a bakers' yeast process in the medium scale vessel is simulated. The experimental data [Noorman et al., 1993b] on the biomass concentration after 8 hours of fermentation, $C_{X}=8 \cdot 16[\mathrm{~g} / \mathrm{l}]$, is used as input in the sugar consumption in Table 2. Other kinetic parameters used, are the specific consumption rate of sugar, $\mathrm{q}_{\mathrm{SMAX}}=2.8[\mathrm{gS} / \mathrm{gX}, \mathrm{h}]$, and a saturation constant of $\mathrm{K}_{\mathrm{S}}=0.18[\mathrm{~g} / \mathrm{l}]$. The concentration of the sugar feed was $610[\mathrm{~g} / \mathrm{l}]$ and the feed rate was $\mathrm{Q}_{s}=2 \cdot 10^{-6}\left[\mathrm{~m}^{3} / \mathrm{s}\right]$. For a completely mixed fermentation, the above information would 


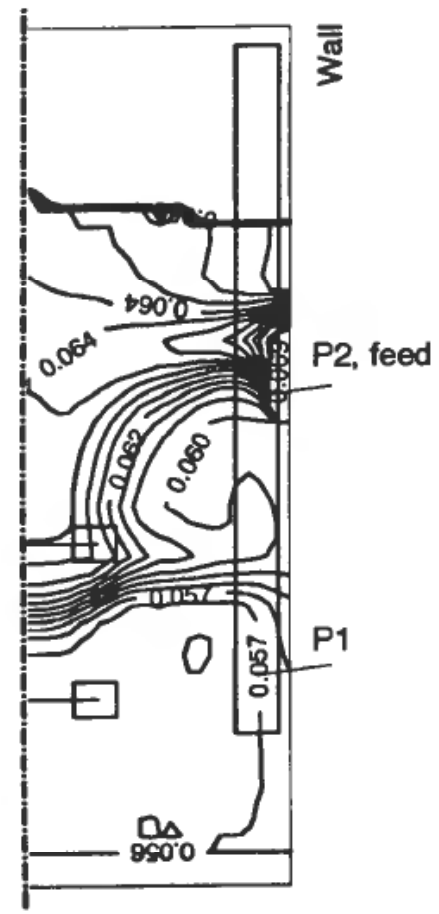

Figure 15. Simulated sugar concentrations in vessel Il at time $=8[\mathrm{~h}] . \mathrm{Q}=1 \cdot 0 \mathrm{VVM}$, $\mathrm{N}=300$ RPM.

result in a sugar concentration at time $=8[\mathrm{~h}]$ of $\mathrm{C}_{\mathrm{S}}=[\mathrm{g} / \mathrm{l}]$. In Figure 15, the predicted sugar concentrations at times $=8[\mathrm{~h}]$ in vessel II are shown. The concentration is seen to be highest in the upper part of the vessel, where the feed is located. At the lower port, $\mathrm{P} 1$, the simulated and measured concentrations were $0.057[\mathrm{~g} / \mathrm{l}]$ and $0.029[\mathrm{~g} / \mathrm{l}]$ respectively, and at the upper part the simulated and measured concentrations were 0.061 and $0.032[\mathrm{~g} / \mathrm{l}]$. Accordingly, both simulations and measurements indicate the highest concentration at the upper port. The simulation gives limited comparisons with experimental data, but they were performed to confirm that the right trends occur concerning the sugar gradients. The two measured concentrations were both lower than what the model predicts for an ideal mixed case.

\section{Large scale vessel}

Tracer response Finally, the simulated and measured tracer response curves for vessel III are shown in Figure 16. The Figure shows normalized concentrations. The measured response shown is an average of four measured responses [Noorman et al., 1993a]. The tracer is injected just below the liquid surface near the shaft, and the detection point is located near the wall at the height of the lower impeller (port PI, Figure 2c)). The simulated curve is seen to respond almost immediately, but to have a much slower $90 \%$ mixing time than found by experiments. The discrepancy between the predicted and measured mixing times is explained by the difference in the total gas holdups. When the gas holdup is large, the tracer will be transported over a larger reactor volume. Moreover, the high gas holdup results in less effective agitation, which will reduce the liquid circulation. 


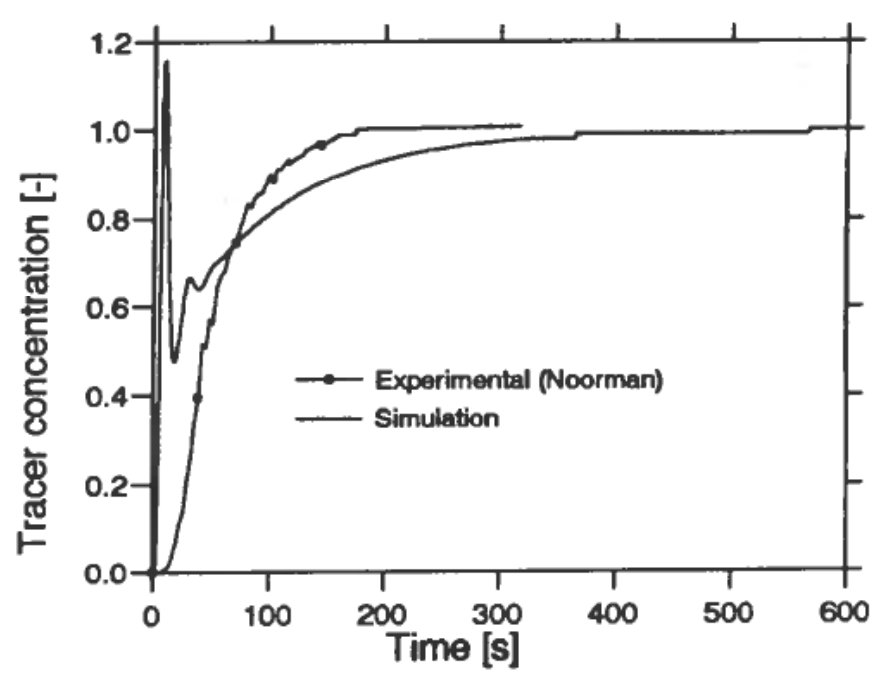

Figure 16. Predicted tracer response curves of vessel III compared against measurements of Noorman et al. (1993a). Q = 0.54 VVM, N = 133 RPM.

\section{Prediction of flow characteristics in isothermal gas/particle fluidized beds}

The model for gas/particle flow given above has been verified in several isothermal circulating fluidized beds by Hjertager and coworkers. A short review of this work will be given here.

\subsection{Mean and turbulence velocity}

Samuelsberg and Hjertager (1996b) have measured and predicted the mean and turbulence velocity profiles in a circulating bed of $30 \mathrm{~mm}$ in diameter, $1 \mathrm{~m}$ in height. They used Laser doppler anemometry to measure the flow velocities.

\section{Mean velocity}

As illustrated in Figure 17 the predicted particle velocities have the same general trend as measured. The particles move upwards in the core and downwards near the walls. A behaviour which is typical for core-annulus flow.

For gas superficial velocity $V_{\mathrm{g} \text {, sup }}=0.71 \mathrm{~m} / \mathrm{s}$, Figure 17 shows how the velocity profile compares well with the experimental results in the core of the reactor at all heights. The core annulus flow in the simulations gives the general trend, but a little too high central velocity at the highest level. The downflow near the wall is overpredicted at all heights. The simulations are not quite symmetrical at the two lowest heights. This may be due to the asymmetrical backflow of particles. There is a slight acceleration of the particles throughout the riser, the flow is not fully developed.

\section{RMS velocity}

The RMS velocity is derived from the granular temperature through the relation $V_{\text {RMS }}=(3 \times \Theta)^{1 / 2}$. A comparison between the simulated RMS velocity and the measured RMS velocity is therefore possible. The general trend in the simulations is in relatively good agreement with the experimental results, although the simulations are a little too low at all levels. The simulated profiles are not quite symmetrical for any of the simulations. 


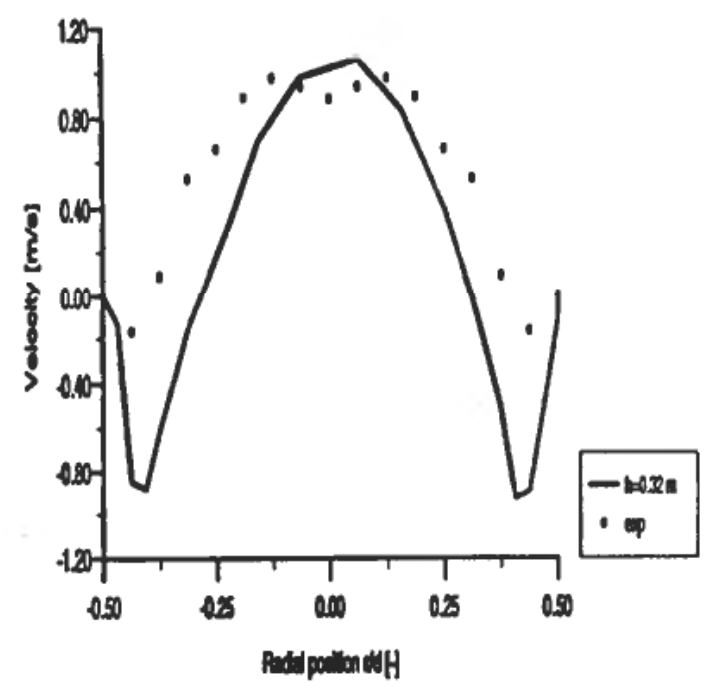

Figure 17. Experimental and simulated particle velocity for $\mathrm{V}_{\mathrm{g}, \text { sup }}=0.71 \mathrm{~m} / \mathrm{s}$.

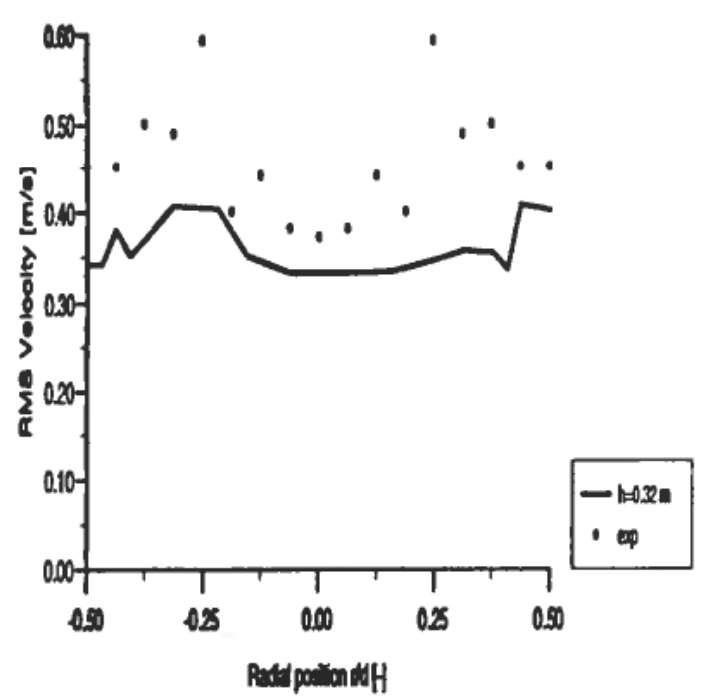

Figure 18. Experimental and simulated particle RMS velocity for $V_{\mathrm{g} \text {, sup }}=0.71 \mathrm{~m} / \mathrm{s}$.

For the case with superficial gas velocity of $0.71 \mathrm{~m} / \mathrm{s}$, it can be seen from Figure 18 , that experimental RMS velocity has the steepest gradients at $0.16 \mathrm{~m}$, and more evenly distributed at the higher levels. This general trend is also given by the simulations, except that the velocities are a little too low.

\subsection{Solids distribution and behaviour}

Samuelsberg and Hjertager (1996c) have predicted the mean velocity and volume fraction profiles in a circulating bed of $75 \mathrm{~mm}$ in diameter, $7 \mathrm{~m}$ in height. The experimental data was provided by Miller and Gidaspow (1992). Mathiesen et al. (1996) have predicted the segregation effect by particle size using four different phases: one for the gas and three for the particles. The geometry was a circulating bed of $200 \mathrm{~mm}$ 
cross section and $2 \mathrm{~m}$ in height. The measured data was taken from Tadrist and Azario (1993).

\section{Solids volume fractions}

Figure 19 shows simulated and experimental solid volume fraction profiles for the case with gas superficial velocity of $2.61 \mathrm{~m} / \mathrm{s}$ and solid flux of $20.4 \mathrm{~kg} / \mathrm{m}^{2} \mathrm{~s}$. At the height of $4.18 \mathrm{~m}$, the core and annulus are correctly predicted as shown in Figure 19. The simulated average volume fractions equals the experimental, which is 0.03 .

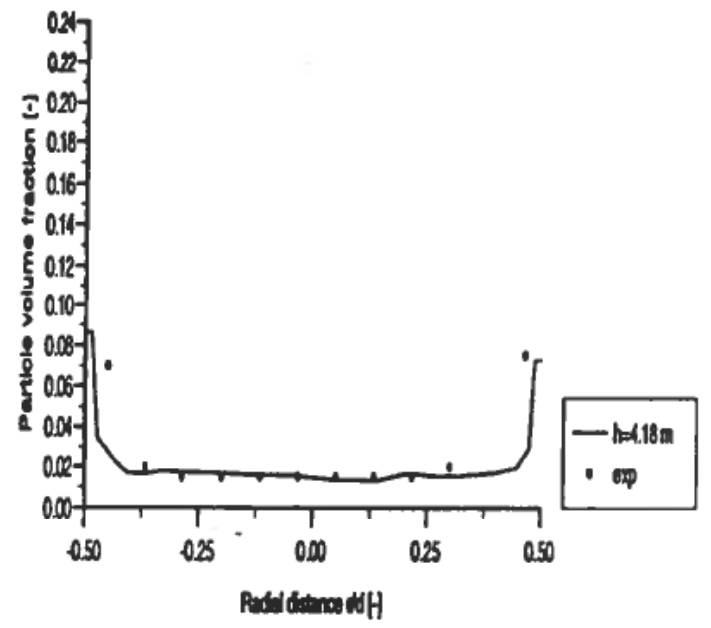

Figure 19. Experimental and simulated particle volume fraction for $C_{p}=20.4 \mathrm{~kg} / \mathrm{m}^{2} \mathrm{~s}$ and $\mathrm{V}_{\mathrm{g}, \text { sup }}=2.61 \mathrm{~m} / \mathrm{s}$.

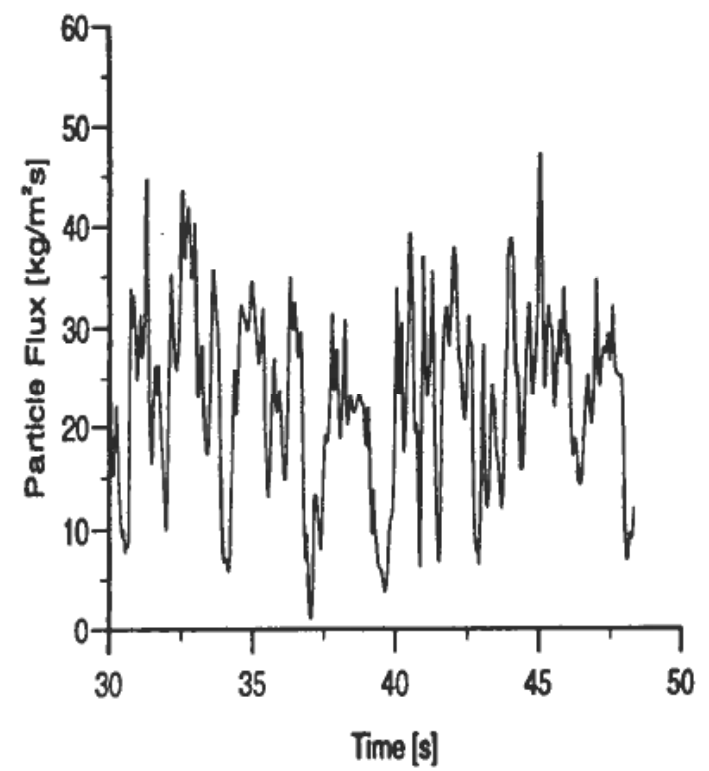

Figure 20. Simulated outlet particle flux for $G_{p}=20 \cdot 4 \mathrm{~kg} / \mathrm{m}^{2} \mathrm{~s}$ and $\mathrm{V}_{\mathrm{s}, \text { sup }}=2 \cdot 61 \mathrm{~m} / \mathrm{s}$. 


\section{Outlet particle flux}

Although the inlet boundary conditions are steady, the flow never reaches a steady state. The gas and the solid will oscillate with a frequency that varies with gas superficial velocity and solid flux. These oscillations increase with increasing riser height and increasing feed flux and will reach a maximum at the top of the riser due to less dampening effect in the upper part. Figure 20 shows the solid mass flux oscillation at the outflowing boundary for the case with particle feed flux of $20.4 \mathrm{~kg} / \mathrm{m}^{2} \mathrm{~s}$ and gas superficial velocity of $2.61 \mathrm{~m} / \mathrm{s}$. The slugs that could be observed visually are also predicted in the simulations. The mean flux of these slugs can grow to more than three times the inlet mass flux.

\section{Particle segregation}

Figure 21 shows the mean particle diameter and standard deviation along the centre line of the reactor. The mean diameter is somewhat overpredicted, but the general trend seems to be well predicted. The figure shows that the multiphase model can predict the segregation effect by size. The predicted standard deviation is in good agreement with the measured one.
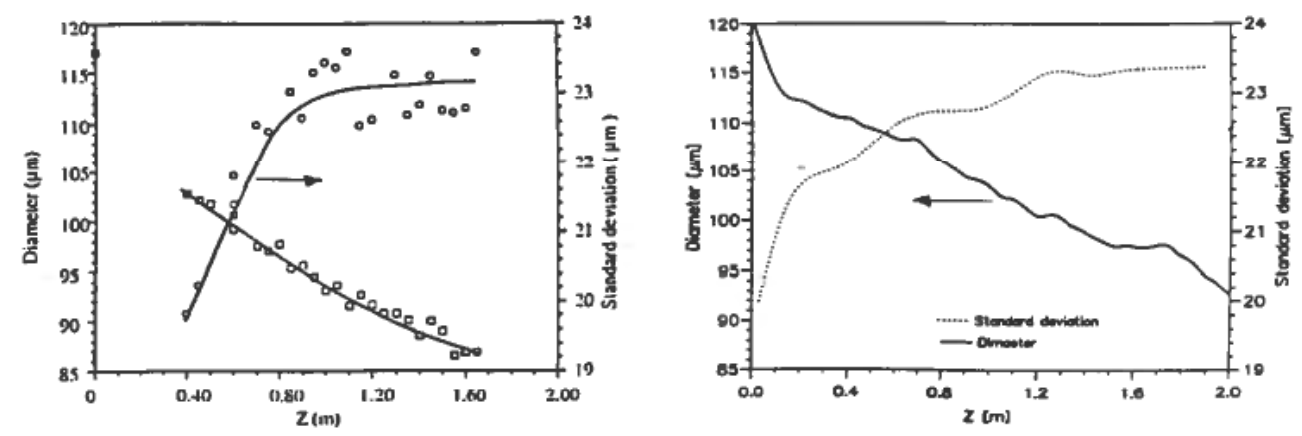

Figure 21. Measured (left) and predicted (right) particle mean and standard deviation of the diameter as function of height (Tadrist and Azario (1993)) for $\mathrm{V}_{\mathrm{g} \text {, sup }}=0.95 \mathrm{~m} / \mathrm{s}$.

\subsection{Species concentration distribution}

Samuelsberg and Hjertager (1995) have predicted the isothermal ozone decomposition in a circulating bed of $250 \mathrm{~mm}$ in diameter, $11 \mathrm{~m}$ in height. The experimental data was provided by Ouyang et al. (1993).

\section{Radial ozone concentration profiles}

Radial profiles of measured and simulated dimensionless ozone concentration at 4 different axial locations are shown in Figure 22. Both measurements and simulations give the same trend, the ozone concentration is higher in the centre than close to the reactor walls. This is consistent with the observed solid volume fractions profiles, since the conversion is directly proportional to the solid volume fraction. At the highest axial position, the profile is almost flat, the difference in ozone concentration between core and wall region decreases with increasing height. The simulated results fit the experimental results well. At the lowest position, the lowest ozone concentration is found experimentally. That cannot be correct and Ouyang claims that entrance effect and mixing of fresh catalysts have influenced the result. Potter (1993) indicated that the 

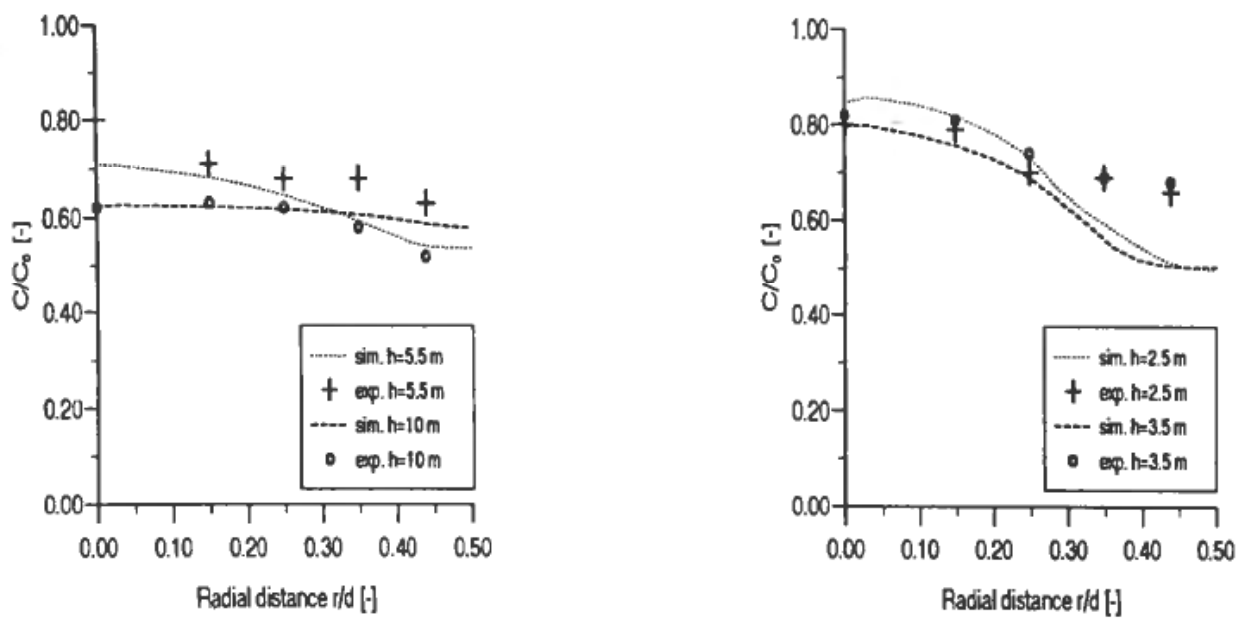

Figure 22. Measured and predicted dimensionless ozone concentration in the upper (left) and middle parts of the riser as function of radial distance.

measured result at this level could be discarded. Comparisons at the lowest level is therefore not given.

\section{Prediction of oxychlorination reaction in a gas particle fluidized bed}

The present example is taken from Samuelsberg and Hjertager (1996a) and will be presented in somewhat more detail than the above gas/particle cases.

\subsection{Initial and boundary conditions}

A simplified oxy-chlorination reaction is simulated in a full-scale bubbling bed reactor to demonstrate the model's capabilities and possibilities to simulate gas/solid flows with exothermic chemical reaction and heat transfer between the phases as well as to internal heat transfer equipment. The reactor is 4 metres in diameter and 20 metres high. It is simulated using a non-uniform Cartesian mesh with $42 \times 68$ gridnodes in the radial and axial direction respectively. Initially the reactor is filled with an 8 metre high bed of FCC catalyst with particle diameter of $60 \cdot 10^{-6} \mathrm{~m}$ and density of $1600 \mathrm{~kg} / \mathrm{m}^{3}$. The inlet gas superficial velocity is held constant at $0.4 \mathrm{~m} / \mathrm{s}$, at the outlet boundary a continuation condition is used for the gas phase. No particles are allowed to leave the reactor. The inlet mass fractions of the chemical species participating in the reaction are the same as the initial mass fraction in the reactor, their values are given in Figure 23. The initial temperature for the gas and solid present in the reactor is the same as the inlet gas temperature which equals $200^{\circ} \mathrm{C}$. The reactor gauge pressure is set to 5 bar. The reaction heat in this strongly exothermic reaction is removed by generation of steam in submerged heat exchangers. The internal heat transfer equipment, which will be hundreds of submerged tubes in a commercial reactor, is here modelled as a porous block with a volume blockage of 0.75 , this is approximately corresponding to 200-250 pipes with a diameter of $0 \cdot 15 \mathrm{~m}$. The temperature in the porous block is held constant at $200^{\circ} \mathrm{C}$, and both phases are able to exchange heat with the heat exchange device. An operating gauge pressure of $150-500 \mathrm{kPa}$ with a temperature of $220-235^{\circ} \mathrm{C}$ in the reactor are typical for the oxy-chlorination reaction in a commercial bubbling 


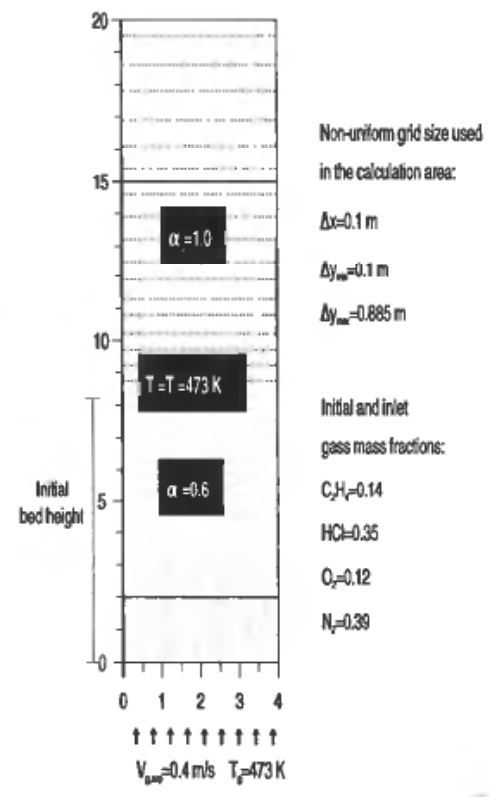

Figure 23. Geometry, boundary and initial conditions.

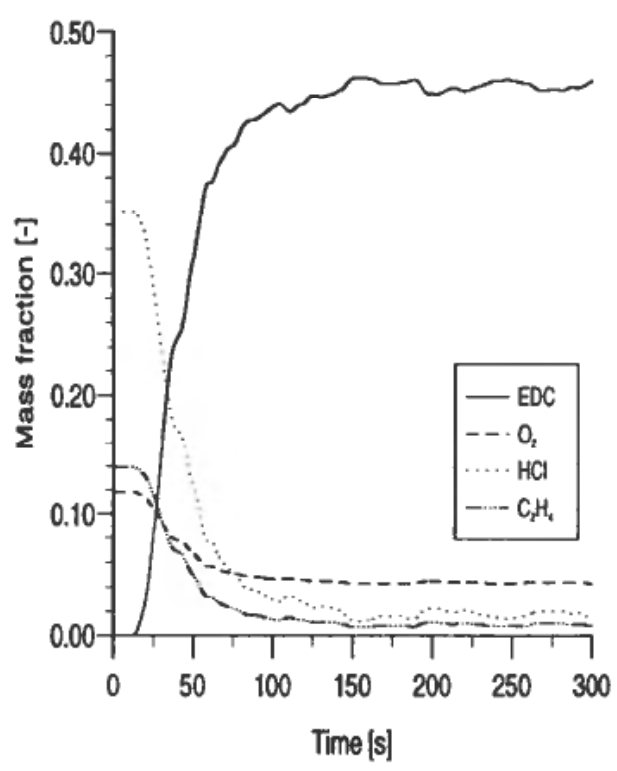

Figure 24. Mass fractions at the outlet as function of time.

fluidized bed reactor (Cowfer and Magistro, 1983). The reactor layout for the simulation of the reactor is given in Figure 23.

\subsection{Chemical reaction and conversion}

In Figure 24, the outlet concentrations of the reactants and the main product are monitored at the outlet. After 25 seconds of real simulation time, the produced EDC has reached the outlet boundary and after approximately 150 seconds, a statistically steady reaction state is reached. From a conversion based on the limiting reactant a steady rate of reaction can be computed. From this reaction rate, the outlet mass fraction of EDC is calculated to be 0.468, which is in good accordance with the result in Figure 24. Even though a steady reaction rate can be computed, large fluctuations in the reaction rate is simulated as shown in Figure 25. The main part of the reaction occurs in the lower uncooled part of the reactor which is a turbulent and transient zone causing good mixing of the reactants. In this highly turbulent section bubbles are formed which makes high and low density zones in the bottom region. In the high density zone, the reaction rate will be high and the conversion takes place instantaneously. In the low density zone, the particle concentration is close to zero and the reaction rate is correspondingly low. As a result, unreacted reactant follows the bubbles through the bed and due to the turbulent gas particle mixing throughout the bed, the conversion zone is not limited to the lower part of the reactor. Close to the wall were the catalyst fraction is high, the gas phase consists mainly of inerts and EDC due to the circulation pattern, thereby the reaction rate is very low in these regions. Due to this axial transport, two distinct reaction rate gradients exist, a radial from the middle of the bed to the walls and one axial, which has a maximum close to the bottom and decreases through the bed. The same pattern will also be found in the temperature due to the tight coupling between reaction rate and temperature. 

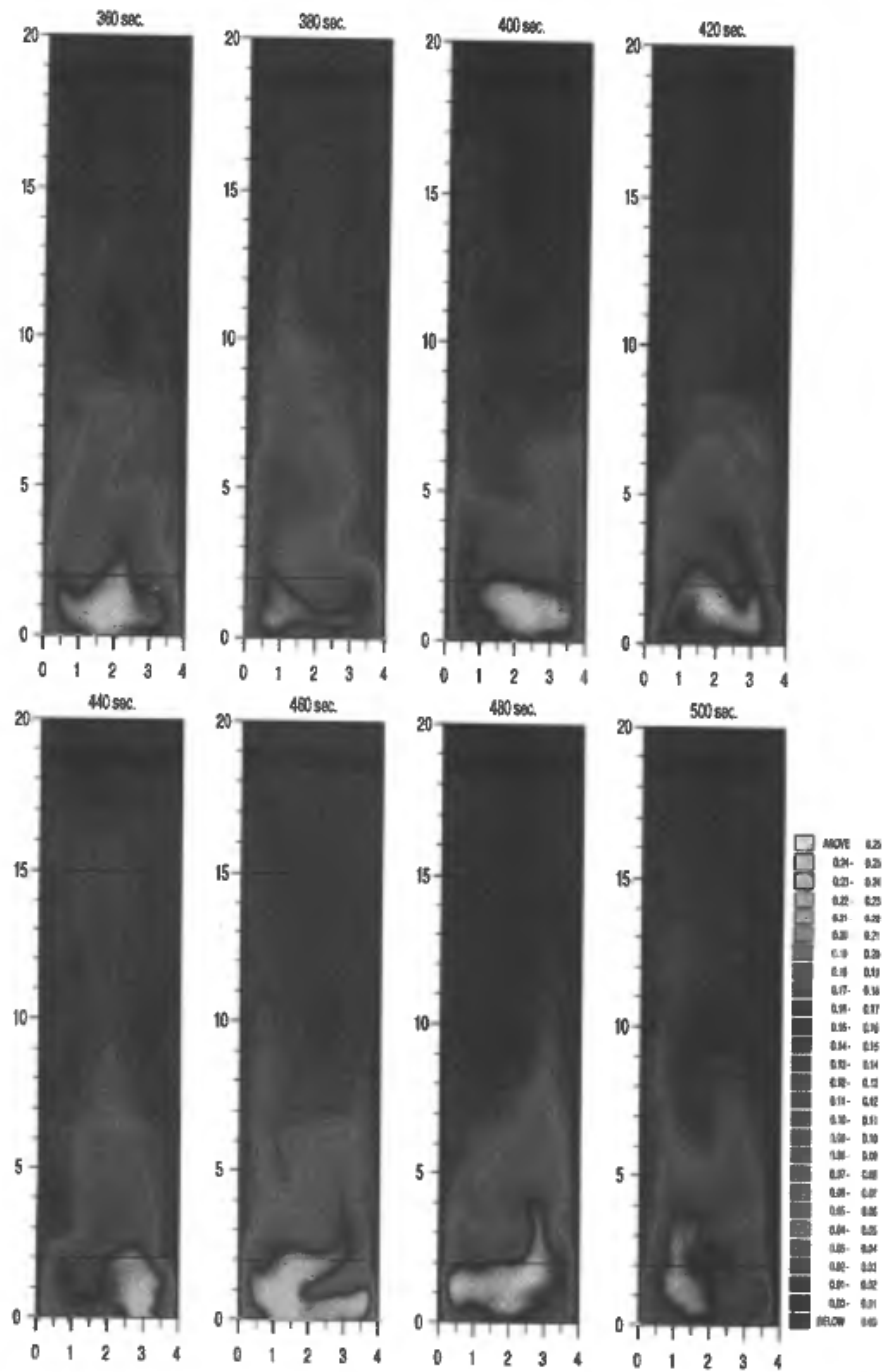

Figure 25. Time series of reaction rate.

\subsection{Temperature distribution in the bed}

In a highly exothermic reaction, temperature control is essential. Too high temperatures will lead to increased by-product formation. As seen in Figure 25, the main part of the reaction occurs in the bottom section where there is no cooling present and thereby the maximum temperature will be present in this section. The temperature difference between the phases present in the reactor is negligible due to the very high heat transfer coefficient.

In Figure 26, the transient gas temperature 0.5 metres above the inlet in the middle of the reactor and at the outlet is shown. In the lower position, the temperature fluctuations are very high, with a maximum temperature of $280^{\circ} \mathrm{C}$ and a minimum of $220^{\circ} \mathrm{C}$. The frequency is $0.11 \mathrm{~Hz}$ which gives a period of 8.9 seconds. This is approximately the same period as for the gas velocity. At the outlet, the oscillations are 


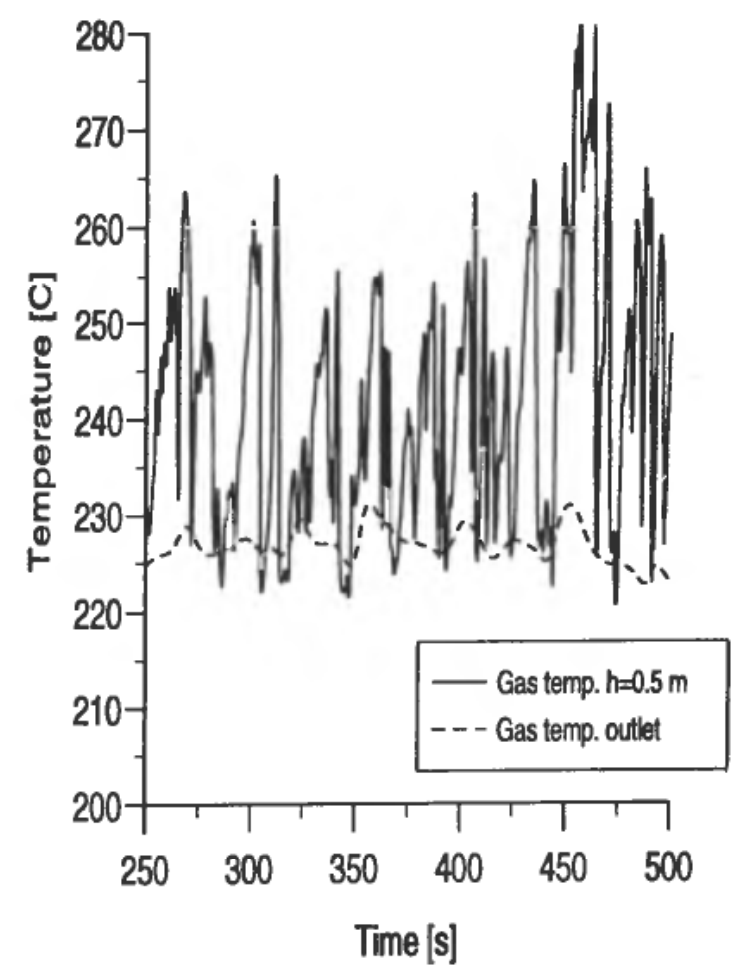

Figure 26. Transient gas temperature in the reactor.

almost negligible with a low frequency of $0.003 \mathrm{~Hz}$, which gives a period of oscillation of 30 seconds. This indicates that there exists an intense turbulent mixing in the lower part which decreases with height.

The time averaged axial gas temperature 0.5 metre from the left and right wall together with gas temperature in the middle of the reactor is shown in Figure 27. There is a very high axial gradient the first half metre. Almost all the temperature rise occurs here. From 2 metres above the grid where the cooling section starts and 10 metres up in the reactor, the temperature decreases until it reaches what will be approximately the outlet temperature. In this zone the highest temperature is in the middle section, which is not surprising since Figure 25 shows a small reaction rate in this section. The temperature difference between left and right in the bed is negligible. In the last 8 metres of the reactor, the temperature is almost constant, the average outlet temperature is $226^{\circ} \mathrm{C}$.

In Figure 28, a time series of the gas temperature is shown. The very high temperature in the bottom section follows the reaction rate and tongues of hot gas are penetrating deep into the bed. Colder sections of gas are found close to the walls, giving high radial temperature gradients in the bottom section. The temperature fluctuations in the upper part can also be seen, where high temperature in the first pictures is displaced by colder gas in the last pictures. From gas flow pattern analyses, it can be seen that sections with high gas circulation give cold zones and sections where the gas flows directly through the bed gives locally high temperature zones. 


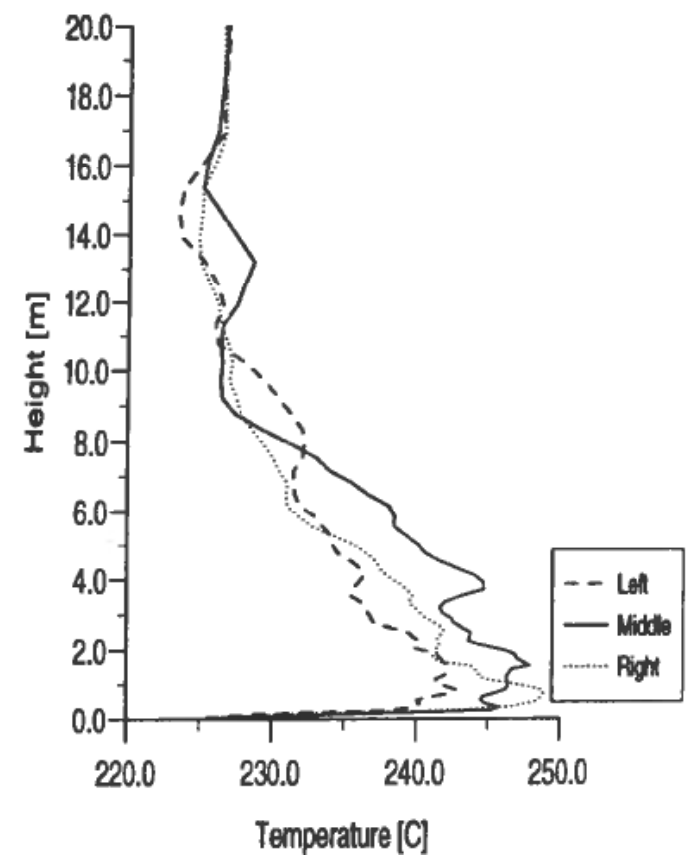

Figure 27. Time averaged axial gas temperature in three radial positions in the reactor.

\subsection{Discussion}

The integrated flow, reaction and heat transfer model is capable of simulating the formation and propagation of gas bubbles in a full scale bubbling bed reactor. The gas and solid circulation pattern set up by these bubbles influence the gas-solids temperature and the chemical species distribution in the bed.

The exothermic reaction model is capable to predict the formation and destruction of the chemical species involved in the chemical reaction. An overall steady reaction state is gained after 120 seconds of real simulation. A conversion based on the limiting reactant of 0.974 is achieved, which gives an outlet mass fraction of EDC of 0.47.

The largest part of the chemical reaction is shown to be occurring in the first metres above the inlet. The large amount of heat generated in this reaction is effectively cooled with the aid of a submerged heat exchange device, modelled as a porous block. The time averaged temperature in the bed ranges from $250^{\circ} \mathrm{C}$ just above the inlet to a temperature of $230^{\circ} \mathrm{C}$ at the outlet boundary. Due to the very large transfer coefficient between the phases involved, the temperature difference between the phases is negligible.

The zones close to the wall is not participating in the reaction and are only used in axial transport of cooler gas and particles from the upper part of the reactor to the lower part. This transport increases the temperature gradients and improve the cooling of the reactor walls, even though the reactor volume must be larger for the reaction only, due to this recirculation. Already produced EDC recirculates unnecessary by the axial transport back to the high temperature zone which may accelerate by-product formation and occupies reactor space.

A more optimal reactor design with the aid of Computational Fluid Dynamics modelling may reduce the reactor size without decreasing the production formation rate or increasing the heat load to the walls. 

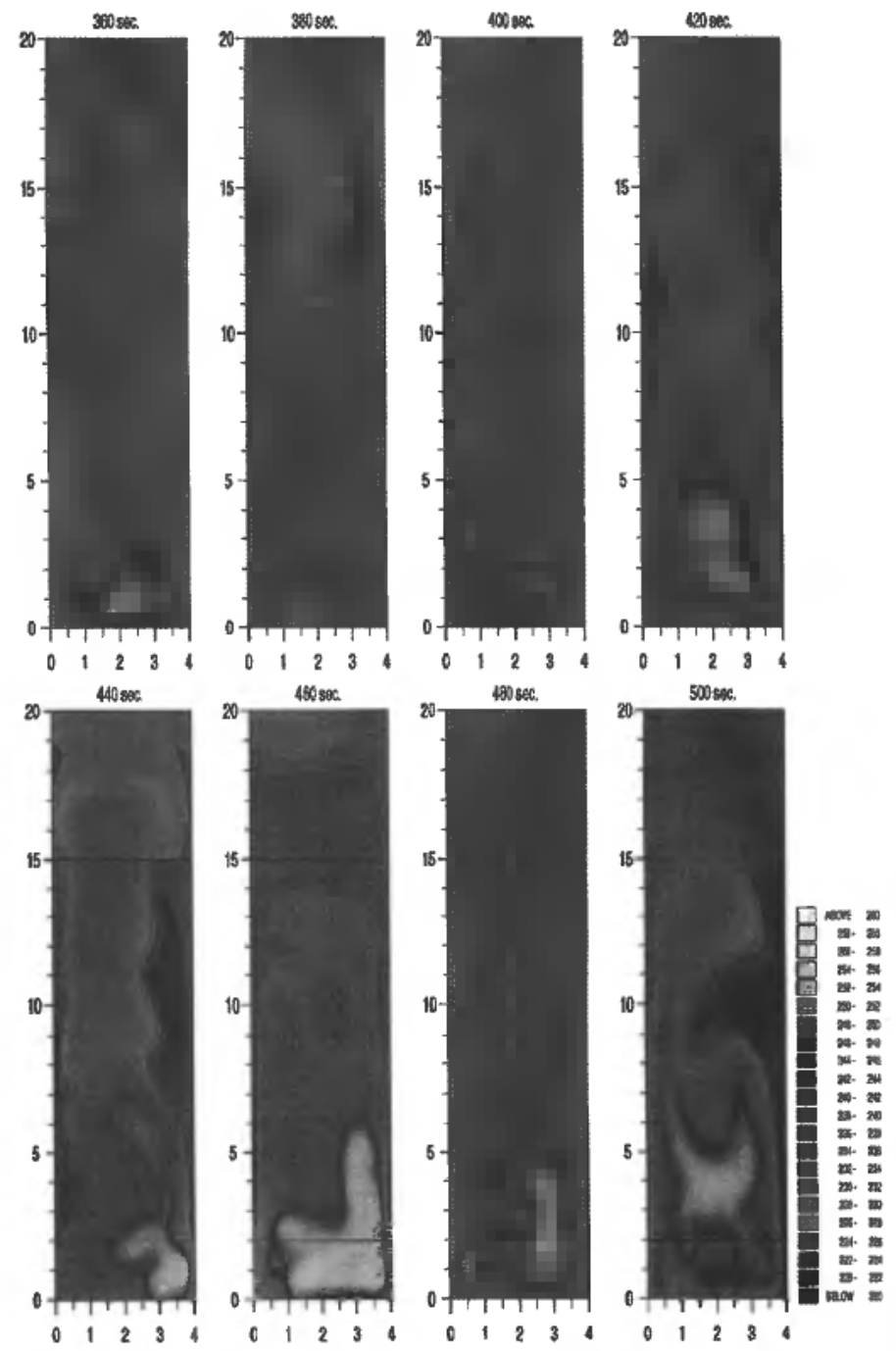

Figure 28. Time series of the gas temperature.

\section{Concluding remarks}

Gas Liquid A computation method for turbulent two-phase flow and reaction kinetics in $2 \mathrm{D}$ bioreactors has been presented. Experimental and computational results of flow pattern and biochemical reaction in three different stirred vessels $\left(15\right.$ litre, $\left.1 \mathrm{~m}^{3}, 30 \mathrm{~cm}^{3}\right)$ have been presented. The agreement between measurements and predictions are in general good.

The kinetic model should be modified to take into account the oxygen limitation locally in the reactor. This requires modelling of the oxygen mass transfer.

The effect of the hydrostatic pressure on the gas-liquid flow in large scale reactors 
should be investigated. Future challenges are modelling and experimental verification of non-newtonian flows and coalescence and breakup of bubbles.

The geometry modelling may be improved by extending the two-fluid model to three dimensions. A three dimensional model allows proper treatment of asymmetric flows like in stirred vessels with baffles.

Further improvements may be introduced by using non-uniform grid spacing in the region near the gas inlet, near the impeller and baffle region for the stirred tank. A better resolution in areas where the gradients are large, without a substantial increase of the number of control volumes, is beneficial. Also, the shape of the gas inlet for the stirred vessel may be improved by introducing a point source instead of having the inlet at the bottom.

Gas particle A comprehensive multidimensional CFD model for turbulent gas particle flow, heat transfer and chemical reaction in fluidized bed reactors is presented. The conservation equations for the solid phase are based on kinetic theory for granular flow based on the work of Jenkins and Savage (1983), Lun et al. (1984) and further extended by Ding and Gidaspow (1990) and Gidaspow (1994). This model makes it possible to calculate a granular temperature and, based on this granular temperature, a solid phase shear viscosity is explicitly computed. The model is incoroprated in a 2-dimensional computer code.

The flow model is compared with available experimental data from literature in a dense Circulating Fluidized Bed riser and from LDA measurements in a dilute laboratory scale circulating fluidized reactor. The turbulent kinetic energy model is capable to reasonable predict oscillations in the solid phase. The velocity profiles are not influenced by varying the restitution coefficient, but the RMS velocity profiles are dramatically changed. The flow model is also capable to predict segregation using four phases: one for the gas and three for three particle sizes. The chemical reaction model is compared with experimental reaction data from an ozone decomposition reaction in a pilot scale CFB. In order to indicate the model's capabilities to simulate the various processes that occur in a fluidized bed reactor, a simplified oxy-chlorination reaction is simulated in a full scale bubbling bed. The chemical reaction and heat transfer model is capable to predict temperature distribution in both gas and solid phase and the formation and destruction of the chemical components involved in the chemical reaction.

Due to the discrepancies found between the experimental and the simulated results, and to be able to simulate commercial processes for reliable scale-up or optimization purposes, further improvements of the model are needed. The recommendations for further work can be summarized as follows:

The flow model should be extended to three dimensions to make the model able to capture three-dimensional effects in order to obtain more detailed information of the processes involved. The model for granular temperature should be extended to include the influence of a multi-sized particle mixture on solid rheology. The chemical reaction model should be extended in order to account for heterogeneous and/or side-reactions in a fluidized bed reactor. The model for mass and heat transfer should be further developed and tested against available data. In order to obtain sharp interfaces, higher order discretizations scheme should be incorporated. Further testing and verification of the combined flow, chemical reaction and heat transfer model against reliable experimental data is vital for the use and acceptance of such models in both research and industrial practice. 


\section{ACKNOWLEDGEMENTS}

The work on bioreactor modelling have been supported by a grant from the Nordic Programme on Bioprocess Engineering under the auspices of NI, the Nordic Fund for Technology and Industrial Development. The present work on bioreactors at HiT-TF/Tel-Tek is sponsored by the Commission of the European Union. The work on fluidized bed reactor modelling is sponsored by the Norwegian Research Council (NFR), Norsk Hydro and Statoil. The author is grateful to Dr. K. Morud and Dr. A. Samuelsberg former $\mathrm{PhD}$ students as well as Mr. V. Mathiesen, $\mathrm{PhD}$ student at Telemark Institute of Technology and Dr. T. Solberg, Senior Scientist and Dr. E. Manger, Scientist at Tel-Tek that all have contributed to the work.

\section{REFERENCES}

Amarasooriya, W. H. and Theofanous, T. G. (1988). Premixing of Steam Explosions. A Three-fluid Model, Preprint, University of California, Santa Barbara.

BAKKER A. and VANDEN AKKER, H. E. (1991). A computational study on dispersing gas in a stirred reactor. Proceedings of the 7th European Congress on Mixing, pp. 199-208.

BAKKER, A. (1992). Hydrodynamics of Stirred Gas-Liquid Dispersions, PhD Thesis, Delft University of Technology, The Netherlands.

BOYSAN, F. et al. (1988). The growth of Cathanranthus roseus in stirred tank bioreactors. In Bioreactor Fluid Dynamics (Elsevier Applied Science Publ.), pp. 245-258.

BRÖRING, S., FISCHER, J., KORTE, T., SOLLINGER, S. and LÜBBERT, A. (1991). Flow Structure of the Dispersed Gasphase in Real Multiphase Chemical Reactors Investigated by a New Ultrasound-Doppler Technique. Canadian J. Chem. Eng., 69, 1247-1256.

CHAPMAN, S. and CoWLING, T. G. (1970). The mathematical theory of non-uniform gases, Third edition. Cambridge University Press.

COWFER, J. A. and MAGISTRO, A. J. (1983). Vinyl chloride, Encyclopedia of chemical technology, Third Edition, Vol. 23, J. Wiley \& Sons, pp. 865-885.

DAVIDSEN, M. (1991). Analysis of flow pattern in a bubble column, MSc Thesis (in Norwegian), Telemark Institute of Technology.

DEARDORFF, J. W. (1971). On the magnitude of the Sub Grid Scale Eddy Coefficient. Journal of Computational Physics, 7, 120-133.

DING, J. and GIDASPOW, D. (1990). A bubbling fluidization model using kinetic theory of granular flow. AIChE Journal, 36, 523-538.

DREw, D. A. (1992). Analytical Modeling of Multiphase Flows. In R. T. Lahey, Jr., editor, Boiling Heat Transfer (Elsevier Science Publishers), pp. 31-84.

ELLuL, I. R. and ISSA, R. I. (1987). Prediction of the Flow of Integrated Gas and Liquid Phases through Pipe Bends. Chem. Eng. Res. Des., 65, 84-96.

ENFors, S. O., GEORGE, S. and LarSon, G., KTH (1992). Private communications.

FISCHER, J., BRÖRING, S. and LÜBBERT, A. (1992). Gas-Phase Properties in Stirred Tank Bioreactors. Chem. Eng. Technol., 15, 390-394.

GELDART, D., editor, (1986). Gas Fluidization Technology.

GeORGE, S., LaRSSON, G., ENFors, S. O., MORUD, K. and HJerTaGer, B. H. (1992). Large-scale test of hydrodynamic-microbial model predictability and comparison with scale down reactor. $215 \mathrm{~m} 3$ bubble column at Svenska Jästfabrik AB, Sollentuna, Sweden, Joint interim report Tel-Tek/KTH.

GIDASPOW, D. (1986). Hydrodnamics of fluidization and heat transfer: Super-computer modeling. Appl. Mech. Rev., 39 (1), 1-23.

Gidaspow, D., ETTEHADIEH, B. and BoullLARD, J. (1985). Hydrodynamics of fluidization: Bubbles and gas compositions in the U-gas process. AIChE Symposium Series, 80 (241), $57-64$.

GIDASPOW, D. and THERDTHIANWONG, A. (1993). Hydrodynamics \& $\mathrm{SO}_{2}$ sorption in a CFB loop. Proceedings of the 4th International Conference on circulating fluid beds, pp. 351-358.

GIDASPOW, D. (1994). Multiphase flow and fluidization, continuum and kinetic theory descriptions, Academic Press.

GoSMAN, A.D.,LEKAKOU, C., POLITIS. S., ISSA, R. I. and LOONEY, M. K. (1992). Multidimensional 
Modeling of Turbulent Two-Phase Flows in Stirred Vessels. AIChE Journal, 38 (12), 1946-1956.

HARVEY, P. S. and GREAVES, M. (1982). Turbulent flow in an agitated vessel. Trans. Inst. of Chem Eng., 60, 195-210.

HueRTAGER, B. H. (1986). Three-Dimensional Modeling of Flow, Heat Transfer, and Combustion. In Handbook of Heat and Mass Transfer (Houston, Gulf Publishing Company), pp. 1303-1350.

HJertager, B. H. (1993). Numerical Analyses of Multiphase Flows, Lecture Notes, Telemark Institute of Technology, Porsgrunn.

HJERTAGER, B. H. and SAMUELSBERG, A. (1992). Computational simulation of flow processes in fluidized bed reactors. KONA Powder and Particle, 10, 96-103.

HJertager, B. H. and Morud, K. (1993). Computational fluid dynamics simulation of bioreactors. Proceedings of Bioreactor Performance Symposium, 15-17 March, Helsingør, Denmark, pp. 47-61.

HJERTAGER, B. H. and MORUD, K. (1993). Multi-dimensional computer modelling of two-phase flow processes in bioreactors. Proceedings of Modelling for Improved Bioreactor Performance, 27-28 September, Bratislava, Slovakia, pp. 13-18.

Huertager, B. H. and Morud, K. (1995). Computational fluid dynamics simulation of bioreactors. Modelling, Identification and Control, 16 (4), 177-191.

Hudcova, V., Machon, V. and NIENOW, A. W. (1989). Gas-Liquid Dispersion with Dual Rushton Turbine Impellers. Biotechnology and Bioengineering, 34, 617-628.

ISSA, R. I. and GOSMAN, A. D. (1981) The computation of three-dimensional turbulent two-phase flows in mixer vessels. In Numerical methods in laminar and turbulent flow, pp. 827-838.

IsHII, M. and ZuBER, N. (1979). Drag Coefficient and Relative Velocity in Bubbly, Droplet or Particulate Flows. AIChE Journal, 25 (5), 843-855.

JAKOBSEN, H. A. (1993). On the Modelling and Simulation of Bubble Column Reactors using a Two-fluid Model, Dr. ing. Thesis, Norwegian Institute of Technology, Norway.

JENKINS, J. T. and SAVAGE, S. B. (1983). A theory for the rapid flow of identical, nearly elastic. spherical particles. Journal of Fluid Mechanics, 130, 187-202.

JU, S. Y., MULVAHILL, T. M. and PIKE, R. W. (1990). Three-dimensional turbulent flow in agitated vessels with a nonisotropic viscosity turbulence model. Canadian J. Of Chem. Eng., 68, 3-16.

KRESTA, S. M. and WoOD, P. (1991). Prediction of the three-dimensional turbulent flow in stirred tanks. AIChE Journal, 37 (3), 448-460.

LAI, K. Y. and SALCUdEAN, M. (1987) Computer analysis of multi-dimensional turbulent buoyancy-induced two-phase flows in gas-agitated reactors. Computers and Fluids, 15 (3), 218-295.

LAHEY, R. T., JR. (1992). The Prediction of Phase Distribution and Separation Phenomena using Two-fluid Models. In Boiling Heat Transfer (Elsevier Science Publishers B.V.), pp. 85-122.

LAUNDER, B. F. and SPALDING, D. B. (1974). The Numerical Computation of Turbulent Flows. Computer Methods in Applied Mechanics and Engineering, 3, 269-289.

Lopez DE Bertodano, M., LeE, S-J., LAHEy, R. T. JR. and Drew, D. A. (1990) The Prediction of Two-Phase Distribution Phenomena Using a Reynolds Stress Model. Journal of Fluids Engineering, 112, 107.

LOPEZDE BERTODANO, M., LAHEY, R. T., JR. and JONES, O. C. (1994). Development of a k- $\varepsilon$ Model for Bubbly Two-phase Flow. Transactions of the ASME, 116, 128-134.

Lun, C. K. K., Savage, S. B., JefFrey, D. J. and Chepurniy, N. (1984). Kinetic theories for granular flow: inelastic particles in Couette flow and slightly inelastic particles in a general flowfield. Journal of Fluid Mechanics, 140, 223-256.

MA, D. and AHMADI, G. (1990a). A thermodynamical formulation for dispersed multiphase turbulent flows-1. Int. Journal of Multiphase Flows, 16 (2), 323-340.

MA, D. and AHMADI, G. (1990b) A thermodynamical formulation for dispersed multiphase turbulent flows-Part II: Simple shear flows for dense mixtures. Int. J. Multiphase Flows, 16, 342-351.

MahmoUdi, S. M. and YiAnNeSKIS, M. (1991). The Variation of Flow Pattern and Mixing Time with Impeller Spacing in Stirred Vessels with two Rushton Impellers, 7th European Congress on Mixing, Brugge, pp. 17-24.

Manger, E., Solberg, T., HJerTaGer, B. H. and VAReide, D. (1995a) Numerical simulation of the ticking hour glass. Int. J Multiphase Flow, 21 (4), 561-567.

MANGER, E., SOLBERG, T. and HJERTAGER, B. H. (1995b) Numerical simulation of a vertical lifter. 
Proceedings of the 5th International Conference on Bulk Materials Handling and Transportation, Newcastle, N.S.W., Australia, 9-12 July, Vol. 1, pp. 199-204.

MANGER, E. (1996a) Modelling and simulation of gas/particle flow using curvilinear coordinates, Dr. ing. Thesis, Telemark Institute of Technology, Porsgrum, Norway.

MANGer, E., Sol berG, T. and HJERTAGer, B. H. (1996b) Simulation of dense particle flow in a CFB riser. 5th International Conference on circulating fluidized beds, May 28-31, Beijing, China.

Mathiesen, V., Solberg, T., Manger, E. and Huertager, B. H. (1996). Modelling and predictions of multiphase flow in a pilotscale circulating fluidized bed. 5th International Conference on circulating fluidized beds, May 28-31, Beijing, China.

Miller, A. and GidASPOw, G. (1992) Dense, vertical gas-solid flow in a pipe. AIChE Journal, 38 (11), 1801-1815.

Migdal, D. and AGOSTA, V. D. (1967). A source flow model for continuum gas-particle flow. Appl. Mech., 35, 860-865.

MoruD, K. E. (1994). Turbulent Two-phase Flow in Bubble Columns and Stirred Fermenters, Dr. ing. Thesis, Telemark Institute of Technology, Porsgrunn, Norway.

Morud, K., SolberG, T. and HJerTAGER, B. H. (1991). Turbulent two-phase air water flow in a bubble column. Paper $X$ in Proceedings of the Bioprocess Engineering meeting in Sandnes, 2-4 April, Biotechnology Research Foundation, Lund, Sweden.

Morud, K. and HJERTAGER, B. H. (1992). Multi-dimensional modelling of processes in bioreactors: Flow and biochemical reaction in a bubble column and flow in a stirred vessel. Proceedings of the Bioprocess Engineering meeting in Stockholm, pp. 40-57, Biotechnology Research Foundation, Lund, Sweden.

MORUD, K. and HJERTAGER, B. H. (1996). LDA measurements and CFD modelling of gas-liquid flow in a stirred vessel. Chemical Engineering Science, 51 (2), 233-249.

Norman, H., Enfors, S.-O., HJerTaGer, B. H., LaRSSON, G., MORUd, K., TrÄGÅRDH. C. and TøRNKVIST, M. (1993a) Verification of integrated microbial and fluid dynamics: Saccharomyces cerevisiae production on $30 \mathrm{~m}^{3}$ scale. In Progress in Biotechnology, editors: Alberghina, Frontali, Sensi, Elsevier Publisher, Vol. 9, Part II, pp. 935-938, 1994.

Norman, H., MORUD, K., HJertager, B. H., TräGÅRDH, C., LARSSON, G. and ENFORS, S.-O. (1993b) CFD modelling and verification of flow and conversion in a $1 \mathrm{~m}^{3}$ bioreactor. $3 \mathrm{rd}$ International Conference on Bioreactor and Bioprocess Fluid Dynamics, 14-16 September, Cambridge, England, pp. 241-258, 1993.

Norman, H., HJertager, B. H., Morud, K., Trăgårdh, C., Enfors, S.-O., Larsson, G. and TÖRNKVIST, M. (1993c) Measurements and CFD Simulation of sacchromyces cervisiae Production in a $30 \mathrm{~m}^{3}$ Stirred Tank Reactor. Proceedings of Bioreactor Performance Symposium, 15-17 March, Helsingør, Denmark, pp. 243-259.

OuYANG, S., Lin, J. and POTTER, O. E. (1993a). Ozone decomposition in a $0.254 \mathrm{~m}$ diameter circulating fluidized bed reactor. Powder Technology, 74, 73-78.

OUYANG, S. and POTTER, O. E. (1993b). Modelling of chemical reaction in a $0.254 \mathrm{~m}$ i.d. circulating fluidized bed. Proceedings of the 4th International conference on circulating fluid beds, pp. 422-427.

POTTER, O. E. (1993). Private communication.

PATANKar, S. V. (1980). Numerical Heat Transfer and Fluid Flow, McGraw-Hill.

Patankar, S. V. and Spalding, D. B. (1972). A Calculation Procedure for Heat, Mass and Momentum Transfer in Three-dimensional Parabolic Flows. Int. Journal of Heat and Mass Transfer, 15, 1787-1800.

Patterson, G. K. (1991). Measurements and Modelling of Flow in Gas Sparged, Agitated Vessels. 7th European Congress on Mixing, Brugge, pp. 209-215.

PlaCEK, J. AND TAVLARIDES, L. L. (1985) Turbulent Flow in Stirred Tanks, Part I: Turbulent Flow in the Turbine Impeller Region. AIChE Journal, 31 (7), 1113-1120.

PlaceK, J., TAVlarides, L. L., SMITH, G. W. and ForT, I. (1986) Turbulent flow in stirred tanks Part II: A two-scale model of turbulence. AIChE Journal, 32 (11), 1771-1786.

Pericleous, K. A. and PATEL, M. K. (1987). The source and sink approach in the modelling of stirred reactors. PCH PhysicoChemical Hydrodynamics, 12, 279-297.

RANADE, V. V. and JoSHI, J. B. (1990). Flow generated by a disc turbine: Part Il-Mathematical modelling and comparison with experimental data. Trans IChemE, 68 (A), 34-50.

RODI, W. (1984). Examples of Turbulence-model Applications. In Turbulence Models and their Applications, Vol. 2, Paris, pp. 295-401. 
SAMUELSBERG, A. (1994). Modelling and simulation of fluidized bed reactors, Dr. ing. Thesis, Telemark Institute of Technology, Porsgrunn, Norway.

SAMUel SBERG, A. and HJerTAGER, B. H. (1995) Simulation of two-phase gas/particle flow and ozone decomposition in a 0.25 m I.D. riser. In Advances in Multiphase Flow-1995 (Amsterdam, Elsevier), pp. 679-688.

SAmuelsberG, A. and HJertager, B. H. (1996a). Computational fluid dynamic simulation of an oxy-chlorination reaction in a full-scale fluidized bed reactor. 5th International Conference on circulating fluidized beds, May 28-31, Beijing, China.

SAMUELSBERG, A. and HJerTAGer, B. H. (1996b). An experimental and numerical study of flow patterns in a circulating fluidized bed reactor. Int. J. Multiphase flow, 22 (3), 575-591.

SAmUelsBerg, A. and HJerTAGer, B. H. (1996c). Computational modelling of gas particle flow in a riser. AIChE Journal, 42 (6), 1536-1546.

Smith, T. J. and ReILLY, C. D. (1988). Predictions of the flow in fermentors and implications for scale-up. In Bioreactor Fluid Dynamics (Elsevier Applied Science Publ.), pp. 431-441.

SChWARZ, M. P. and TURNER, W. J. (1988). Applicability of the Standard k- $\varepsilon$ Turbulence Model to Gas Stirred Baths. Appl. Math Modelling, 12.

SHIH, Y. T., GidASPOW, D. and WASAN, D. T. (1987). Hydrodynamics of sedimentation of multisized particles. Powder Technology, 50, 201-215.

SpaldiNG, D. B. (1977). The Calculation of Free-convection Phenomena in Gas-Liquid Mixtures, ICHMT seminar 1976. In Turbulent Buoyant Convection (Hemisphere), pp. 569-586.

Spalding, D. B. (1980). Numerical Computation of Multi-phase Fluid Flow and Heat Transfer, Recent Advances in Numerical Methods in Fluids (Pineridge Press), pp. 139-168.

Spalding, D. B. (1985). Computer Simulation of Two-phase Flows with Special Reference to Nuclear Reactor Systems. In R. W. Lewis, K. Morgan, J. A. Johnson and W. R. Smith, editors, Computational Techniques in Heat Transfer (Pineridge Press), pp. 1-44.

TADRIST, L. and AZARIO, E. (1993). Analyses of two-phase flow in a circulating fluidized bed: Local measurements using Phase Doppler analyser. Proceedings of the 4th International Conference on Circulating fluidized beds.

THEOLOGOS, K. N. and MARKATOS, N.C. (1992). Modelling of flow and heat transfer in fluidized catalytic cracking riser-type reactors. Trans. IChemE, 70 (A), 239-245.

TheOLOGOS, K. N. and MARKATOS, N. C. (1993). Advanced modelling of fluid catalytic cracking riser-type reactors. AIChE Journal, 39 (6), 1007-1017.

Takeda, H., Narasaki, K., Kitajima, H., Sudoh, S., ONofusa, M. and Iguchi, S. (1993). Numerical Simulation of Mixing Flows in Agitated Vessels with Impellers and Baffles. Computers Fluids, 22 (2/3), 223-228.

TORVIK, R. and SVENDSEN, H. F. (1990). Modelling of slurry reactors. A fundamental approach. Chem. Eng. Sci., 45 (8), 2325-2332.

Tsuo, Y. P. and GiDASPOW, D. (1990). Computations of flow-patterns in circulating fluidized beds. AIChE Journal, 36, 885-896.

TRÄGÅRDH, C. (1988). A hydrodynamic model for the simulation of an aerated agitated fed-batch fermentor. In Bioreactor Fluid Dynamics (Elsevier Applied Science Publ.), pp. 117-134.

TRÄGÅRDH, C., HEINZLE, E. and SANER, E. U. (1990). Mathematical modelling of mixing and biokinetics in agitated tank fermentors. Proceedings of the Fifth European Conference on Biotechnology, pp. 797-800.

VAN SWAAIJ, W. P. M. and ZUIDERWEG, F. J. (1972). Investigation of ozone decomposition in fluidized beds on the basis of a two-phase model. Proceedings of the European Symposium on Chemical reaction engineering, pp. B 9-25-36.

\section{Nomenclature}

$A_{b} \quad$ volume averaged surface area of baffles

$\mathrm{A}_{\mathrm{f}} \quad$ volume averaged surface area of paddles

$a_{i} \quad$ specific interfacial surface area

B width of impeller blade

c constants

C drag coefficient or concentration

C Ozone concentration 
$\mathrm{C}_{0} \quad$ Ozone concentration at the inlet boundary

$\mathrm{C}_{\text {out }} \quad$ Ozone concentration at the outlet boundary

$\mathrm{c}_{\mathrm{p}} \quad$ specific heat capacity

$\mathrm{D}_{\mathrm{i}} \quad$ impeller diameter

D tank diameter

$\mathrm{d}_{\mathrm{p}} \quad$ particle diameter

e coefficient of restitution

$\mathrm{F} \quad$ flow rate

$\mathrm{FL}_{\mathrm{g}} \quad$ flow number, $\mathrm{Q}_{\mathrm{g}} / \mathrm{nD}^{3}{ }_{\mathrm{i}}$

$\overrightarrow{\mathrm{F}}_{\mathrm{k}} \quad$ interface friction force

$\vec{g} \quad$ gravitational acceleration vector

$\mathrm{g}_{\mathrm{j}} \quad \mathrm{j}$-direction component of gravity

$\mathrm{g}_{0} \quad$ radial distribution function

$\mathrm{G}$ production of turbulent kinetic energy

$h_{v} \quad$ volumetric heat transfer coefficient between the phases

$h_{w} \quad$ volumetric bed to wall heat transfer coefficient

h enthalpy

$\Delta \mathrm{H}_{\mathrm{rx}} \quad$ standard heat of reaction

$\overrightarrow{\text { J }} \quad$ scalar flux vector

k specific reaction rate constant

$\mathrm{k}_{\mathrm{g}} \quad$ gas laminar conductivity

k turbulent kinetic energy

$\mathrm{K}_{\mathrm{s}} \quad$ saturation constant for substrate

$\mathrm{K}_{\mathrm{se}} \quad$ saturation constant for ethanol

$\mathrm{n}$ name of baffles or paddles or RPM

$\mathrm{N}_{\mathrm{p}} \quad$ gas-particle Nusslet number

p pressure

$\mathrm{P}_{\mathrm{b}} \quad$ production of turbulent kinetic energy due to bubbles

$\mathrm{q}_{\mathrm{s}} \quad$ spec. substrate concentration rate

$\mathrm{q}_{\mathrm{smax}}$ max. spec. substrate concentration rate

qsxa substrate composition rate, aerobe

$\mathrm{q}_{\mathrm{sxf}} \quad$ substrate consumption rate, anaerobe

$\mathrm{q}_{\mathrm{ep}} \quad$ ethanol production rate

$\mathrm{q}_{\mathrm{ec}} \quad$ ethanol consumption rate

$\mathrm{q}_{\mathrm{o}} \quad$ total oxygen consumption rate

qos oxygen consumption rate

qomax max. spec. oxygen concentration rate

$\mathrm{Q}_{\mathrm{g}} \quad$ gas flow rate

$\mathrm{Re} \quad$ Reynolds number

$\mathrm{r}^{*} \quad$ dimensionless radius $(2 \mathrm{r} / \mathrm{D})$

S source term

t time

$T_{g}, T_{p} \quad$ temperature of gas, solid

$T_{w} \quad$ and wall, respectively

$\mathrm{U}, \mathrm{V}$ mean velocity components

V volume

$\vec{V}_{\mathrm{g}}, \vec{V}_{\mathrm{p}}$ gas and solids velocity vector

$\overrightarrow{\mathrm{V}} \quad$ velocity vector 


$\begin{array}{ll}\mathrm{Y} & \text { mass fraction } \\ \mathrm{Y}_{\mathrm{os}} & \text { yield gO/gS } \\ \mathrm{Y}_{\mathrm{es}} & \text { yield } \mathrm{gE} / \mathrm{gS} \\ \mathrm{Y}_{\mathrm{oe}} & \text { yield gO/gE } \\ \mathrm{Y}_{\mathrm{xsa}} & \text { yield } \mathrm{gX} / \mathrm{gS} \text {, aerobic } \\ \mathrm{Y}_{\mathrm{xsf}} & \text { yield } \mathrm{gX} / \mathrm{gS} \text {, anaerobic } \\ \mathrm{Y}_{\mathrm{xe}} & \text { yield } \mathrm{gX} / \mathrm{gE}\end{array}$

\section{Greek letters}

$\begin{array}{ll}\alpha & \text { void fraction } \\ \beta & \text { two phase drag coefficient } \\ \gamma & \text { collisional energy dissipation } \\ \delta_{\mathrm{ij}} & \text { kroenecker delta } \\ \Theta & \text { turbulent kinetic energy or granular temperature } \\ \kappa & \text { thermal conductivity } \\ \mu & \text { Shear viscosity } \\ \pi & 3 \cdot 14 \\ \mathrm{p} & \text { density } \\ \xi & \text { solid bulk viscosity } \\ \tau & \text { stress tensor gas phase } \\ \varepsilon & \text { dissipation of turbulent kinetic energy } \\ \Phi & \text { general variable } \\ \mu & \text { viscosity or specific growth rate } \\ \underline{\sigma} & \text { shear stress tension }\end{array}$

\section{Subscripts}

$\begin{array}{ll}\mathrm{g} & \text { gas phase } \\ \mathrm{i} & \text { inlet } \\ \mathrm{k} & \text { phase number } \\ \mathbf{1} & \text { liquid } \\ \mathrm{o} & \text { outlet } \\ \mathrm{p} & \text { particle } \\ \mathrm{t} & \text { turbulent } \\ \mathrm{S} & \text { solid }\end{array}$

\section{Abbreviations}
2-D Two-dimensional
LDA Laser doppler anemometer
PDA Phase doppler anemometer
RMS Root mean square
RPM Revolutions per minute
VVM Volume gas per volume liquid and minute 